Groups Geom. Dyn. 6 (2012), 485-531

DOI $10.4171 / \mathrm{GGD} / 164$
Groups, Geometry, and Dynamics

(C) European Mathematical Society

\title{
Cohomology computations for Artin groups, Bestvina-Brady groups, and graph products
}

\author{
Michael W. Davis* and Boris Okun
}

\begin{abstract}
We compute:
- the cohomology with group ring coefficients of Artin groups (or actually, of their associated Salvetti complexes), of Bestvina-Brady groups of type FP, and of graph products of groups,

- the $L^{2}$-Betti numbers of Bestvina-Brady groups of type FP over $\mathbb{Q}$, and of graph products of groups,

- the weighted $L^{2}$-Betti numbers of graph products of Coxeter groups.
\end{abstract}

In the case of arbitrary graph products there is an additional proviso: either all factors are infinite or all are finite.

Mathematics Subject Classification (2010). 20F36, 20F55, 20F65, 20J06, 55N25; 20E42, $57 \mathrm{M} 07$.

Keywords. Artin group, Bestvina-Brady group, building, Coxeter group, graph product, right-angled Artin group, $L^{2}$-Betti number, weighted $L^{2}$-cohomology.

\section{Contents}

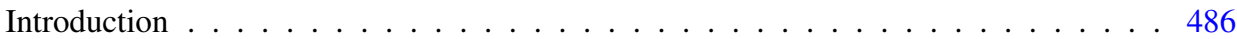

1 Basic definitions . . . . . . . . . . . . . . . . . . . . . . 490

2 A spectral sequence . . . . . . . . . . . . . . . . . . . . 496

3 Some previous cohomology computations . . . . . . . . . . . 500

4 New computations . . . . . . . . . . . . . . . . . . . 503

5 Graph products of Coxeter groups . . . . . . . . . . . . . . . . . . . 509

6 Weighted $L^{2}$-cohomology of buildings and Coxeter groups $\ldots \ldots \ldots$. . . . . . . . 514

7 Weighted $L^{2}$-Betti numbers of graph products of Coxeter groups . . . . . . . . . 518

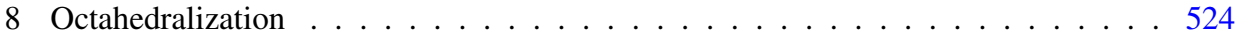

9 Duality groups . . . . . . . . . . . . . . . . . . . . . . 528

References . . . . . . . . . . . . . . . . . . . . 530

*Partially supported by NSF grants DMS 0706259, DMS 1007068. 


\section{Introduction}

This paper concerns the calculation of the group cohomology, $H^{*}(G ; N)$, for certain discrete groups $G$, where the $G$-module $N$ is either $\mathbb{Z} G$ or a von Neumann algebra $\mathcal{N}(G)$. Here $\mathcal{N}(G)$ is a completion of the group algebra $\mathbb{R} G$ acting on $L^{2}(G)$, the Hilbert space of square summable functions on $G$. If $G$ acts properly and cocompactly on a $\mathrm{CW}$ complex $Y$, then one has the reduced $L^{2}$-cohomology spaces, $L^{2} H^{*}(Y)$. These are Hilbert spaces with orthogonal $G$-actions. As such, each has a "von Neumann dimension" with respect to $\mathcal{N}(G)$. The dimension of $L^{2} H^{n}(Y)$ is the $n^{\text {th }} L^{2}$-Betti number, $L^{2} b^{n}(Y ; G)$. When $Y$ is acyclic, it is an invariant of $G$, denoted $L^{2} b^{n}(G)$. As shown in [30], these $L^{2}$-Betti numbers can be computed from the cohomology groups $H^{*}(G ; \mathcal{N}(G))$ (i.e., the cohomology of $B G$ with local coefficients in $\mathcal{N}(G)$ ).

For Coxeter groups, there is a refinement of the notion of $L^{2}$-Betti number. Suppose that $(W, S)$ is a Coxeter system and $\boldsymbol{p}$ is a multiparameter of positive real numbers (meaning that $\boldsymbol{p}$ is a function $S \rightarrow(0, \infty)$ which is constant on conjugacy classes). There is an associated "Hecke-von Neumann algebra", $\mathcal{N}_{p}(W)$, which one can use to define the "weighted $L^{2}$-Betti numbers", $L_{\boldsymbol{p}}^{2} b^{n}(W)$, cf. [21], [15] or [11].

Associated to $(W, S)$, there is a finite CW complex $X$, called its Salvetti complex. It is homotopy equivalent to the quotient by $W$ of the complement of the (possibly infinite) complex hyperplane arrangement associated to $(W, S)$ (cf. [9]). The fundamental group of $X$ is the Artin group $A$ associated to $(W, S)$. The $K(\pi, 1)$-Conjecture for Artin groups asserts that $X$ is a model for $B A(=K(A, 1))$. This conjecture is known to hold in many cases, for example when $W$ is either right-angled or finite, cf. [8].

Given a simplicial graph $\Gamma$ with vertex set $S$ and a family of groups $\left\{G_{s}\right\}_{s \in S}$, their graph product, $\prod_{\Gamma} G_{s}$, is the quotient of the free product of the $G_{s}$ by the relations that elements of $G_{s}$ and $G_{t}$ commute whenever $\{s, t\}$ is an edge of $\Gamma$. Associated to $\Gamma$ there is a flag complex $L$ with 1-skeleton $\Gamma$. ( $L$ is defined by the requirement that a subset of $S$ spans a simplex of $L$ if and only if it is the vertex set of a complete subgraph of $\Gamma$.) A right-angled Coxeter group $W_{L}$ (a RACG for short) is a graph product where each $G_{s}$ is cyclic of order 2. Similarly, a right-angled Artin group $A_{L}$ (a RAAG for short) is a graph product where each $G_{s}$ is infinite cyclic. An arbitrary graph product of groups acts simply transitively on the set of chambers of a rightangled building of type $\left(W_{L}, S\right)$. In $\$ 5.2$ we consider a family of arbitrary Coxeter systems $\left\{\left(V_{s}, T_{S}\right)\right\}_{s \in S}$. Their graph product, $V:=\prod_{\Gamma} V_{s}$, gives a Coxeter system $(V, T)$ (where $T$ denotes the disjoint union of the $T_{s}$ ).

Given a right-angled Artin group $A_{L}$, let $\pi: A_{L} \rightarrow \mathbb{Z}$ be the homomorphism which sends each standard generator to 1 . Define the Bestvina-Brady group, $\mathrm{BB}_{L}$, to be Ker $\pi$. In [4] Bestvina and Brady prove that $\mathrm{BB}_{L}$ is type FP if and only if the simplicial complex $L$ is acyclic.

A subset of $S$ (the fundamental set of generators of a Coxeter group $W$ ) is spherical if it generates a finite subgroup of $W$. Let $\delta(W, S)$ denote the poset of spherical 
subsets of $S$ and let $K$ be the geometric realization of this poset. For each spherical subset $J$, let $K_{J}$ (resp. $\left.\partial K_{J}\right)$ be the geometric realization of the subposet $\delta(W, S)_{\geq J}$ (resp. $\left.\delta(W, S)_{>J}\right) . K_{J}$ is the cone on $\partial K_{J}$. ( $\partial K$ is the barycentric subdivision of the nerve of $(W, S) ; \partial K_{J}$ is the barycentric subdivision of the link of the simplex corresponding to $J$ in the nerve.)

Many of the following computations are done by using a spectral sequence associated to a double complex. The $E_{\infty}$-terms of such a spectral sequence compute the graded group, Gr $H^{*}(-)$, associated to the corresponding filtration of the cohomology group, $H^{*}(-)$, in question.

In item (1) below, the Coxeter system is arbitrary while in (2), (3), (4) it is rightangled. (Within parentheses we refer either to the theorem in this paper where the computation appears or else we give a reference to the literature.) Here are the computations.

(1) Suppose that $A$ is the Artin group associated to a Coxeter system $(W, S), X$ is the associated Salvetti complex and $\tilde{X}$ is its universal cover. Then

(a) (Theorem 4.1).

$$
\operatorname{Gr} H^{n}(X ; \mathbb{Z} A)=\bigoplus_{J \in \mathcal{S}(W, S)} H^{n-|J|}\left(K_{J}, \partial K_{J}\right) \otimes H^{|J|}\left(A_{J} ; \mathbb{Z} A\right) .
$$

(In the case of a RAAG this formula is the main result of [28].)

(b) ([17], Corollary 2).

$$
L^{2} b^{n}(\tilde{X} ; A)=b^{n}(K, \partial K)
$$

(Here $b^{n}(Y, Z)$ is the ordinary Betti number of a pair $(Y, Z)$, i.e., $b^{n}(Y, Z):=\operatorname{dim} H^{n}(Y, Z ; \mathbb{R})$.)

When the $K(\pi, 1)$-Conjecture holds for $A$, these formulas compute, Gr $H^{*}(A ; \mathbb{Z} A)$ and $L^{2} b^{*}(A)$, respectively.

(2) Suppose that $\mathrm{BB}_{L}$ is the Bestvina-Brady subgroup of a RAAG, $A_{L}$.

(a) (Theorem 4.3). Suppose that $L$ is acyclic. Then the cohomology of $\mathrm{BB}_{L}$ with group ring coefficients is isomorphic to that of $A_{L}$ shifted down in degree by 1 :

$$
\operatorname{Gr} H^{n}\left(\mathrm{BB}_{L} ; \mathbb{Z} \mathrm{BB}_{L}\right)=\bigoplus_{J \in \mathcal{S}(W, S)} H^{n-|J|+1}\left(K_{J}, \partial K_{J}\right) \otimes \mathbb{Z}\left(A_{L} / A_{J}\right) .
$$

(b) (Theorem 4.4). Suppose that $L$ is $\mathbb{Q}$-acyclic (where $\mathbb{Q}$ denotes the rational numbers). Then the $L^{2}$-Betti numbers of $\mathrm{BB}_{L}$ are given by

$$
L^{2} b^{n}\left(\mathrm{BB}_{L}\right)=\sum_{s \in S} b^{n}\left(K_{s}, \partial K_{s}\right)
$$


(3) Suppose that $G=\prod_{\Gamma} G_{S}$ is a graph product of groups, and let $(W, S)$ be the RACS associated to the graph. For each spherical subset $J, G_{J}$ denotes the direct product $\prod_{s \in J} G_{s}$.

(a) (Theorem 4.5). If each $G_{S}$ is infinite, then

$$
\operatorname{Gr} H^{n}(G ; \mathbb{Z} G)=\bigoplus_{\substack{J \in \mathcal{S}(W, S) \\ i+j=n}} H^{i}\left(K_{J}, \partial K_{J} ; H^{j}\left(G_{J} ; \mathbb{Z} G\right)\right) .
$$

(b) (Theorem 4.6). If each $G_{S}$ is infinite, then

$$
L^{2} b^{n}(G)=\bigoplus_{\substack{J \in \mathcal{S}(W, S) \\ i+j=n}} b^{i}\left(K_{J}, \partial K_{J}\right) \cdot L^{2} b^{j}\left(G_{J}\right) .
$$

(c) ([13], Corollary 9.4). If each $G_{S}$ is finite, then

$$
\operatorname{Gr} H^{n}(G ; \mathbb{Z} G)=\bigoplus_{J \in S(W, S)} H^{n}\left(K, K^{S-J}\right) \otimes \widehat{A}^{J}
$$

Here $K^{S-J}$ denotes the union of subcomplexes $K_{s}$, with $s \in S-J$, and $\widehat{A}^{J}$ is a certain (free abelian) subgroup of $\mathbb{Z}\left(G / G_{J}\right)$.

(d) ([15], Theorem 13.8, and Corollary 6.5 below). Again, when each $G_{S}$ is finite, $G$ acts simply transitively on the set of chambers of a rightangled building of type $(W, S)$ and its $L^{2}$-Betti numbers are determined by the weighted $L^{2}$-Betti numbers of $(W, S)$ via the formula, $L^{2} b^{n}(G)=$ $L_{\boldsymbol{p}}^{2} b^{n}(W)$, where the multiparameter $\boldsymbol{p}=\left(p_{s}\right)_{s \in S}$ is defined by $p_{s}=$ $\left|G_{s}\right|-1$.

(4) Suppose that $V$ is a graph product of Coxeter groups $\left\{V_{s}\right\}_{s \in S}$, that $(W, S)$ is the RACS associated to the graph and that $\boldsymbol{q}$ is a multiparameter for $(V, T)$. There are the following two results concerning the weighted $L^{2}$-Betti numbers of $(V, T)$.

(a) (Theorem 7.2). Suppose that $\boldsymbol{q}$ is "large" in the sense that it does not lie in the region of convergence for the growth series of any component group $V_{s}$. Then

$$
L_{\boldsymbol{q}}^{2} b^{n}(V)=\sum_{\substack{i+j=n \\ J \in \mathcal{S}}} b^{i}\left(K_{J}, \partial K_{J}\right) \cdot L_{\boldsymbol{q}}^{2} b^{j}\left(V_{J}\right)
$$

(b) (Theorem 7.7). For $V$ as above and $\boldsymbol{q}$ "small" in the sense that it lies in the convergence region of each $V_{s}$, then

$$
L_{\boldsymbol{q}}^{2} b^{*}(V)=L_{\boldsymbol{p}}^{2} b^{*}(W),
$$

where the multiparameter $\boldsymbol{p}$ for $W$ is defined by $p_{s}=V_{s}(\boldsymbol{q})-1$. 
Of course, a graph product $V$ of Coxeter groups is again a Coxeter group. The weighted $L^{2}$-Betti numbers of arbitrary Coxeter groups are computed in [21], [15], but only for the parameter $\boldsymbol{q}$ in a certain specified range: either $\boldsymbol{q}$ or $\boldsymbol{q}^{-1}$ must lie in the closure of the region of convergence for the growth series of the Coxeter group. The point of (4) is that for Coxeter group $V$ which is a graph product, we now have calculations for an extended range of $\boldsymbol{q}$. Thus, in (4a), the formula depends only on the weighted $L^{2}$-Betti numbers of the factors $V_{s}$. (For example, if each $V_{s}$ is an infinite dihedral group these numbers are known for all $\boldsymbol{q}$.) On the other hand, in (4b), the formula for the $L_{\boldsymbol{q}}^{2} b^{*}(V)$ depends only on the weighted $L^{2}$-Betti numbers of the associated RACG, $W$. So, if we happen to know $L_{\boldsymbol{p}}^{2} b^{*}(W)$ for all $\boldsymbol{p}$, we can calculate $L_{\boldsymbol{q}}^{2} b^{*}(V)$ for certain $\boldsymbol{q}$ outside the usual range (cf. Example 8.4).

The calculations in (1), (2), (3a), (3b) and (4a) all follow a similar line. They are based on the spectral sequence developed in $\$ 2$. In all cases we are computing some type of cohomology of a $\mathrm{CW}$ complex $Y$, which is covered by a family of subcomplexes $\left\{Y_{J}\right\}_{J \in S}(W, S)$, indexed by $S(W, S)$. For a fixed $j$, the $E_{1}^{*, j}$-terms of the spectral sequence form a cochain complex for a nonconstant coefficient system on $K$, which associates to a simplex $\sigma$ of $K$ with minimum vertex $J$ the group $H^{j}\left(Y_{J}\right)$. We first show that the spectral sequence decomposes at $E_{1}$ as a direct sum, with a component for each $J \in S(W, S)$, and with the $J$-component consisting of a cochain complex of the form $C^{*}\left(K_{J}, \partial K_{J}\right)$ with some constant system of coefficients. We then show the spectral sequence collapses at $E_{2}$.

Since $\mathbb{Z} G$ is a $G$-bimodule, both sides of the formulas in (1a), (2a) and (3a) are right $G$-modules. One can ask if these formulas give isomorphisms of $G$-modules. The spectral sequence argument shows that this is indeed the case. Moreover, since the right-hand side of each formula is finitely generated as a $G$-module, so is the left-hand side (cf. [14]). In general, if we change the $\mathrm{Gr} H^{n}(-)$ on the left-hand side to $H^{n}(-)$, then these formulas do not give isomorphisms of $G$-modules. For example, (3a) is not valid after this change in the case where $G$ is the free product of two infinite cyclic groups (cf. [14], Example 5.2). However, the possibility remains that after dropping the Gr's, both sides of these equations are still isomorphic as $\mathbb{Z}$-modules, which leads us to the following question.

Question. On the left-hand sides of the formulas in (1a), (2a) and (3a), is Gr $H^{n}(-)$ always isomorphic to $H^{*}(-)$ as an abelian group?

The calculations in (3c), (3d) and (4b) come from a different direction based on [13] and [15]. In particular, the proof of (4b) uses an argument, similar to one in [15], relating the ordinary $L^{2}$-cohomology of a building to the weighted $L^{2}$-cohomology of its Coxeter system.

Our thanks go to the referee for some helpful comments. 


\section{Basic definitions}

1.1. Coxeter groups, Artin groups, buildings. Throughout this paper, we will be given as data a simplicial graph $\Gamma$ with finite vertex set $S$ and edge set, $\operatorname{Edge}(\Gamma)$, together with a labeling of the edges by integers $\geq 2$. The label corresponding to $\{s, t\} \in \operatorname{Edge}(\Gamma)$ is denoted by $m(s, t)$. These data give a presentation of a Coxeter group $W$ with generating set $S$ and relations

$$
s^{2}=1, \quad(s t)^{m(s, t)}=1 \quad \text { for all } s \in S \text { and }\{s, t\} \in \operatorname{Edge}(\Gamma) .
$$

The pair $(W, S)$ is a Coxeter system; $\Gamma$ is its presentation graph. These same data determine a presentation for an Artin group $A$ with generating set $\left\{a_{s}\right\}_{s \in S}$ and relations,

$$
a_{s} a_{t} \ldots=a_{t} a_{s} \ldots \quad \text { for all }\{s, t\} \in \operatorname{Edge}(\Gamma),
$$

where there are $m(s, t)$ terms on each side of the equation. Given a word $s=$ $\left(s_{1}, \ldots, s_{n}\right)$ in $S$, its value is the element $w(s)$ of $W$ defined by

$$
w(s):=s_{1} \ldots s_{n} .
$$

The word $s=\left(s_{1}, \ldots, s_{n}\right)$ is a reduced expression if $s$ is a word of minimum length for its value. The pair $\left(W_{J}, J\right)$ is also a Coxeter system (cf. [11], Theorem 4.1.6). For any $J \subseteq S$, the special subgroup $W_{J}$ is the subgroup generated by $J$. The subset $J$ is spherical if $\left|W_{J}\right|<\infty$. Let $\delta(W, S)$ denote the poset of spherical subsets of $S$. The nonempty elements of $\delta(W, S)$ form an abstract simplicial complex $L(W, S)$, called the nerve of the Coxeter system. $(\operatorname{Vert}(L(W, S))=S$ and a nonempty subset $J \subseteq S$ spans a simplex if and only if it is spherical.)

Given a subset $I \subseteq S$, an element $w \in W$ is $I$-reduced if $l(s w)>l(w)$ for all $s \in I$. For $I \subseteq J \subseteq S$, let $W_{J}^{I}$ be the subset of all $I$-reduced elements in the special subgroup $W_{J}$. (For example, $W_{J}^{\emptyset}=W_{J}$ and $W_{J}^{J}=\{1\}$.)

A chamber system over a set $S$ is a set $\mathcal{C}$ (of "chambers") and a family of equivalence relations $\left\{\sim_{s}\right\}_{s \in S}$ on $\mathcal{C}$ indexed by $S$. (There is one equivalence relation for each $s \in S$.) An $s$-equivalence class is an s-panel. Distinct chambers $C, D \in \mathcal{C}$ are $s$-adjacent if they belong to the same $s$-panel. A gallery in $\mathcal{C}$ is a sequence $\mathbb{C}=\left(C_{0}, \ldots, C_{n}\right)$ of adjacent chambers. Its type is the word $s=\left(s_{1}, \ldots, s_{n}\right)$ in $S$ where the $i^{\text {th }}$ letter of $s$ is $s_{i}$ if $C_{i-1}$ be $s_{i}$-adjacent to $C_{i}$. A building of type $(W, S)$ is a chamber system $\mathcal{C}$ over $S$ equipped with a function $\delta: \mathcal{C} \times \mathcal{C} \rightarrow W$ (called a Weyl distance) such that

(1) Each panel contains at least two elements.

(2) Given a reduced expression $s$ and chambers $C, D \in \mathcal{C}$, there is a gallery of type $s$ from $C$ to $D$ if and only if $\delta(C, D)=w(s)$.

(The above definition of building is due to Ronan and Tits, a variant can be found in [1].) The building $\mathcal{C}$ is locally finite if each panel is finite. 
Example. A Coxeter group $W$ can be given the structure of a chamber system by declaring the $s$-panels to be the left cosets, $w W_{s}$, where $W_{s}\left(=W_{\{s\}}\right)$ is the cyclic group of order two generated by $s$. Define $\delta_{W}: W \times W \rightarrow W$ by $\delta\left(w, w^{\prime}\right)=w^{-1} w^{\prime}$. Then $\left(W, \delta_{W}\right)$ is a building, called the standard thin building of type $(W, S)$.

Given a building $(\mathcal{C}, \delta)$ of type $(W, S)$ and a subset $J$ of $S$, the $J$-residue containing a chamber $C$ is the subset $R_{J}(C) \subset \ell$ defined by

$$
R_{J}(C):=\left\{D \in \mathcal{C} \mid \delta(C, D) \in W_{J}\right\}
$$

In other words, a $J$-residue is a " $J$-gallery connected component of $\varphi$ ". If $J=\emptyset$, then a $J$-residue is simply a chamber and if $J$ has only one element, then a $J$-residue is a panel. In the standard thin building $\left(W, \delta_{W}\right)$, a $J$-residue is a left coset of $W_{J}$.

1.2. Posets, simplicial complexes, flag complexes. Suppose that $L$ is a simplicial complex with vertex set $S$ and let $S(L)$ denote the poset of (vertex sets of) simplices in $L$ (including the empty simplex). If $J$ is the vertex set of a simplex $\sigma$ in $L$, then denote by $\operatorname{Lk}(J, L)$ (or simply $\operatorname{Lk}(J)$ when $L$ is understood), the link of $\sigma$ in $L$. The abstract simplicial complex $\operatorname{Lk}(J)$ has one simplex for each element of $S(L)_{>}$ $\left(=\left\{J^{\prime} \in S(L) \mid J^{\prime} \supset J\right\}\right)$.

A simplicial complex $L$ is a flag complex if any finite, nonempty set of vertices, which are pairwise connected by edges, spans a simplex of $L$. A simplicial graph $\Gamma$ determines a flag complex, $L(\Gamma)$ : its simplices are the finite, nonempty sets of vertices which are pairwise connected by edges.

Suppose that $\mathcal{P}$ is a poset. There is an abstract simplicial complex Flag $(\mathcal{P})$ with vertex set $\mathcal{P}$ and with simplices the totally ordered, finite, nonempty subsets of $\mathcal{P}$. We note that $\operatorname{Flag}(\mathcal{P})$ is a flag complex. Given a simplex $\sigma \in \operatorname{Flag}(\mathcal{P})$, its least element is its minimum vertex and is denoted by $\min (\sigma)$. If $L$ is a simplicial complex, then Flag $\left(S(L)_{>\emptyset}\right)$ can be identified with the barycentric subdivision of $L$. Similarly, Flag $(S(L))$ is the cone on the barycentric subdivision of $L$. (The empty set provides the cone point.)

1.3. Davis complexes and Salvetti complexes. Let $M$ be a topological space. A mirror structure on $M$ over a set $S$ is a family of subspaces $\left\{M_{S}\right\}_{s \in S}$ indexed by $S$; $M_{S}$ is the $s$-mirror of $M$. If $M$ is CW complex and each $M_{S}$ is a subcomplex, then $M$ is a mirrored $C W$ complex. For each $x \in M$,

$$
S(x):=\left\{s \in S \mid x \in M_{s}\right\} .
$$

If $M$ is a mirrored CW complex and $c$ is a cell in $M$, then

$$
S(c):=\left\{s \in S \mid c \subset M_{s}\right\} .
$$

Given a building $\ell$ of type $(W, S)$ and mirrored CW complex $M$ over $S$, define an equivalence relation $\sim$ on $\varphi \times M$ by $(C, x) \sim\left(C^{\prime}, x^{\prime}\right)$ if and only if $x=x^{\prime}$ and 
$C, C^{\prime}$ belong to the same $S(x)$-residue. Give $\mathcal{C}$ the discrete topology, $\mathcal{C} \times M$ the product topology, and denote the quotient space by

$$
\mathcal{B}(\mathcal{C}, M):=(\mathcal{C} \times M) / \sim
$$

and call it the $M$-realization of $\mathcal{C}$. When $\mathcal{C}$ is the standard thin building $W$, put

$$
\mathcal{U}(W, M):=\mathscr{B}(W, M)
$$

and call it the $M$-realization of the Coxeter system $(W, S)$.

Remark. In our previous work, e.g., in [11], we used the notation $U($, , ) to denote a topological realization of either a building or of a Coxeter system. However, in what follows we will study Coxeter systems $(V, T)$ which will also have the structure of a RAB over an auxiliary RACS $(W, S)$ and we will want to distinguish the two types of realizations of $(V, T)$ (either as a Coxeter system or as a building).

The mirror structure is $W$-finite if $S(x)$ is spherical for each $x \in M$. (In this paper we shall always assume this.) When this is the case, $U(W, M)$ is a locally finite complex and similarly, if $\mathcal{C}$ is locally finite building, then $\mathcal{B}(\mathcal{C}, M)$ is a locally finite complex.

We will use the following notation for unions and intersections of mirrors. For any $J \subseteq S$, put

$$
M_{J}:=\bigcap_{s \in J} M_{s}, \quad M^{J}:=\bigcup_{s \in J} M_{s} .
$$

Also, we will write $\partial M_{J}$ for the subset of $M_{J}$ consisting of all points $x \in M$ such that $S(x)$ is a proper subset of $J$.

As in $\S 1.2, \delta(W, S)$ (or simply $\delta$ ) is the poset of spherical subsets of $S$ (including the empty set). The geometric realization of this poset is the simplicial complex Flag $(\mathcal{S}(W, S)$ ). We denote it by $K(W, S)$ (or simply $K$ ) and call it the Davis chamber. Most often we will want to take $M=K$. One gets a mirror structure on $K$ by defining $K_{S}$ to be the geometric realization of the subposet $\delta_{\geq\{s\}}$. Then $U(W, K)$ is the Davis complex and $\mathscr{B}(\mathcal{C}, K)$ is the standard realization of $\mathscr{B}$. By construction, the $W$ action on $\mathcal{U}(W, K)$ is proper (i.e., each isotropy subgroup is finite) and the quotient space $K$ is a finite complex, hence, compact. Moreover, $U(W, K)$ is contractible (by [11], Theorem 8.2.13). Note that for any $J \in S$, the subcomplex $\partial K_{J}$ is the barycentric subdivision $\operatorname{Lk}(J)$ (the link in $L$ of the simplex with vertex set $J$ ). Also, $K_{J}$ is the cone on $\partial K_{J}$ (i.e., $K_{J} \cong \operatorname{Cone}(\operatorname{Lk}(J))$ ).

The Salvetti complex. Let $A$ be the Artin group associated to $(W, S)$ and let $q: A \rightarrow$ $W$ denote the natural homomorphism sending $a_{s}$ to $s$. There is a set-theoretic section for $q$, denoted by $w \mapsto a_{w}$, defined as follows: if $s_{1} \ldots s_{n}$ is any reduced expression for $w$, then $a_{w}:=a_{s_{1}} \ldots a_{s_{n}}$. As explained in [8], p. 602, it follows from Tits' solution to the word problem for Coxeter groups that $w \mapsto a_{w}$ is well defined. 
Define a partial order on $W \times S$ by $(w, I)<(v, J)$ if and only if $I<J$ and $v^{-1} w \in W_{J}^{I}$ (where $W_{J}^{I}$ was defined in $\$ 1.1$ ). It is proved in [9] that $W \times S$ is the poset of cells of a cell complex $X^{\prime}$ on which $W$ acts freely so that each cell of $X^{\prime}$ is a Coxeter cell. ( $\operatorname{Flag}(W \times S)$ is the barycentric subdivision of $X^{\prime}$.) The quotient space $X:=X^{\prime} / W$ is the Salvetti complex of $(W, S)$. It is known that $\pi_{1}(X)=A$. The universal cover of $X$ is denoted by $\tilde{X}$. For each $(w, J) \in W \times S$, the flag complex on $(W \times S)_{\leq(w, J)}$ can be identified with the barycentric subdivision of a Coxeter cell of type $\left(W_{J}\right)$. (A Coxeter cell of type $W_{J}$ means the convex hull of a generic orbit in the canonical representation of $W_{J}$; see [11], §7.3.) So $X^{\prime}$ (or $X$ ) can be given the structure of a CW complex where the cells are Coxeter cells. In particular, each vertex of $X^{\prime}$ corresponds to an element of $W \times S$ of the form $(w, \emptyset)$ and each 1-cell of $X^{\prime}$ corresponds to an element of the form $(w,\{s\})$. Orient this edge by declaring $(w, \varnothing)$ to be its initial vertex and $(w s, \emptyset)$ its terminal vertex. Since the $W$-action preserves edge orientations, these orientations pass to the edges of $X$. Call a vertex $x$ of a cell $C$ of $X$ a top vertex of $C$ if each edge of $C$ which contains $x$ points away from $x$ (cf. [17], §7). One can then explicitly describe CW structure on $X$ as follows. For each $J \in S$, take a Coxeter cell $C_{J}$ of type $W_{J}$. Now for each $I<J$ and each $u \in W_{J}^{I}$ glue together $C_{I}$ and $u C_{I}$ via the homeomorphism induced by $u$. The result is denoted $X_{J}$. (It is the Salvetti complex for $A_{J}$ and therefore, a $K\left(A_{J}, 1\right)$.) To construct $X$, start with the disjoint union of the $X_{J}$, for $J \in S$, and then use the natural maps to identify $X_{I}$ with a subcomplex of $X_{J}$ whenever $I<J$. This description exhibits $X$ as a "poset of spaces over $\delta$ " (as defined in $\S 2$ ). On the level of fundamental groups we know that the inclusion $X_{J} \rightarrow X$ induces the natural injection $A_{J} \rightarrow A$ and that the associated "simple complex of groups" is the one discussed in [8], §3. Similarly, for each $J \in S$, let $\tilde{X}_{J}$ denote the inverse image of $X_{J}$ in $\tilde{X}$. $\left(\tilde{X}_{J}\right.$ is a disjoint union of copies of the universal cover $X_{J}$, one copy for each coset of $A_{J}$ in $A$.) Thus, $\tilde{X}$ also has the structure of a poset of spaces over $S$.

In the right-angled case, the Salvetti complex has a simple description as a subcomplex of a torus (and we will denote it by $T_{L}$ instead of $X$ ). Let $T^{S}$ denote the $S$-fold product of copies of $S^{1}$. Define a subcomplex of $T^{S}$ by

$$
T_{L}:=\bigcup_{J \in \mathcal{S}(L)} T^{J}
$$

(This is a special case of the "polyhedral product" construction discussed in the next section and in [2], [20].) According to [9], its universal cover $\widetilde{T}_{L}$ is a CAT(0)-cubical complex and hence, is contractible.

1.4. Graph products of groups and spaces. As in the Introduction, $\Gamma$ is a simplicial graph and suppose that each edge is labeled 2. Also, $S=\operatorname{Vert}(\Gamma), L$ is the flag complex determined by $\Gamma$ and $\left(W_{L}, S\right)$ is the associated RACS. (We shall generally reserve the notation $W_{L}$ for the case when the Coxeter group is right-angled with nerve $L$ and similarly, $A_{L}$ for the RAAG associated to $L$.) Suppose that $\left\{G_{S}\right\}_{s \in S}$ is a family of groups indexed by $S$. The graph product of the $G_{S}$, denoted $\prod_{\Gamma} G_{S}$, is the 
quotient of the free product of the $G_{s}, s \in S$, by the normal subgroup generated by all commutators of the form, $\left[g_{s}, g_{t}\right]$, where $\{s, t\} \in \operatorname{Edge}(\Gamma), g_{s} \in G_{s}$ and $g_{t} \in G_{t}$. For example, if $\operatorname{Edge}(\Gamma)=\emptyset$, then $\prod_{\Gamma} G_{s}$ is the free product, while if $\Gamma$ is the complete graph on $S$, then $\prod_{\Gamma} G_{S}$ is the direct sum.

Suppose that $G=\prod_{\Gamma} G_{S}$. We want to see that $G$ has the structure of a RAB of type $\left(W_{L}, S\right)$. The group $G$ can be given the structure of a chamber system as follows: the $s$-panels are the left cosets $g G_{s}$, with $g \in G$. Write $G_{S}^{*}$ for $G_{s}-\{1\}$. The projections $G_{s}^{*} \mapsto s$ induce a map (not a homomorphism) $\pi: G \rightarrow W_{L}$, as follows: any element $g \in G$ can be written as $g_{s_{1}} \ldots g_{s_{n}}$, with $g_{s_{i}} \in G_{s_{i}}^{*}$ so that $s_{1} \ldots s_{n}$ is a reduced expression for an element $w \in W$. Moreover, $w$ depends only on $g$. The map $\pi$ sends $g$ to $w$. The Weyl distance $\delta: G \times G \rightarrow W_{L}$ is defined by $\delta(g, h)=\pi\left(g^{-1} h\right)$. The following lemma is easily checked.

Lemma 1.1 ([11], Example 18.1.10). $(G, \delta)$ is a building of type $\left(W_{L}, S\right)$.

Polyhedral products. Suppose, for the moment, that $S$ is the vertex set of an arbitrary simplicial complex $L$. Let $\left\{\left(Z_{s}, A_{s}\right)\right\}_{s \in S}$ be a family of pairs of spaces indexed by $S$. For each $J \in S(L)$, let $Z_{J}^{\prime}$ be the subspace of the product $\prod_{s \in S} Z_{s}$, consisting of all $S$-tuples $\left(x_{s}\right)_{s \in S}$ such that

$$
x_{s} \in \begin{cases}Z_{s} & \text { if } s \in J, \\ A_{s} & \text { if } s \notin J .\end{cases}
$$

The polyhedral product of this family, denoted by $\pi_{L}\left(Z_{S}, A_{S}\right)$, is defined to be the following subspace of $\prod_{s \in S} Z_{s}$ :

$$
\pi_{L}\left(Z_{S}, A_{S}\right):=\bigcup_{J \in \mathcal{S}(L)} Z_{J}^{\prime}
$$

(This terminology comes from [2]. In [20] it is called a "generalized moment angle complex".)

Example 1.2. Suppose that each $\left(Z_{s}, A_{s}\right)=([0,1], 0)$. Then $\pi_{L}([0,1], 0)$ can be identified with Flag $(S(L))$ in such a fashion that a standard subdivision of each cube in the polyhedral product is a subcomplex of Flag $(S(L))$. In particular, if $L$ is the nerve of a RACS, then $\pi_{L}([0,1], 0)=K$, the Davis chamber from $\S 1.3$.

Graph products of spaces. We return to the assumption that $L$ is the flag complex determined by $\Gamma$.

Example 1.3. Suppose that $\left(Z_{s}, A_{s}\right)=\left(\operatorname{Cone}\left(G_{s}\right), G_{s}\right)$ for a family of discrete groups $\left\{G_{s}\right\}_{s \in S}$. The group $G_{s}$ acts on $Z_{s}=\operatorname{Cone}\left(G_{s}\right)$ and $A_{s}=G_{s}$ is an invariant subspace. Let $G^{\prime}:=\prod_{s \in S} G_{S}$ denote the direct product. Then $G^{\prime}$ acts on $\prod_{s \in S} Z_{S}$ and $Z^{\prime}:=\pi_{L}\left(\operatorname{Cone}\left(G_{S}\right), G_{S}\right)$ is an invariant subspace. The quotient space $Z^{\prime} / G^{\prime}$ 
can be identified with the chamber $K=\pi_{L}([0,1], 0)$ of the previous example. It is proved in [12] that the universal cover of $Z^{\prime}$ is the standard realization of a RAB. It follows that the group $G$ of all lifts of elements in $G^{\prime}$ is the graph product, $G=\prod_{\Gamma} G_{s}$. An explanation for this, which is different from that in [12], is given in the following lemma.

Lemma 1.4. With notation as in Example 1.3, the fundamental group of $Z^{\prime}=$ $\pi_{L}\left(\operatorname{Cone}\left(G_{s}\right), G_{s}\right)$ can be identified with the kernel of the natural surjection $G=$ $\prod_{\Gamma} G_{s} \rightarrow G^{\prime}=\prod_{s \in S} G_{s}$. Moreover, if $Z \rightarrow Z^{\prime}$ is the corresponding covering space, then the $G^{\prime}$-action on $Z^{\prime}$ lifts to a $G$-action on $Z$.

Proof. First consider the special case where $S$ consists of two elements $s$ and $t$ and $\Gamma$ has no edges. Then $\operatorname{Cone}\left(G_{s}\right) \times \operatorname{Cone}\left(G_{t}\right)$ is a 2-complex and the polyhedral product $Z^{\prime}$ is the union of 1-cells which do not contain the product of the cone points. Such a 1-cell either has the form $\operatorname{Cone}\left(g_{s}\right) \times g_{t}$ or $g_{s} \times \operatorname{Cone}\left(g_{t}\right)$ for some $\left(g_{s}, g_{t}\right) \in G_{s} \times G_{t}$. These two 1-cells fit together to give a single edge $e\left(g_{s}, g_{t}\right):=$ (Cone $\left.\left(g_{s}\right) \times g_{t}\right) \cup\left(g_{s} \times \operatorname{Cone}\left(g_{t}\right)\right)$ connecting $g_{t}$ to $g_{s}$. In this way we see that $Z^{\prime}$ is identified with (the barycentric subdivision of) the join of $G_{s}$ and $G_{t}$, which we denote $G_{s} \odot G_{t}$. The group $G_{s} \times G_{t}$ acts on $G_{s} \odot G_{t}$ and the vertex stabilizers are either $G_{s}$ or $G_{t}$. The universal cover of $G_{s} \odot G_{t}$ is a tree $T$. The group of all lifts of the $\left(G_{s} \times G_{t}\right)$-action is transitive on edges, and the quotient space is a single edge (with distinct vertices). Hence, the group of lifts is the free product $G_{s} * G_{t}$ and $T$ is the corresponding Bass-Serre tree.

The general case follows in the same manner by considering the universal cover of the 2-skeleton of $Z^{\prime}$.

Next suppose that for each $s \in S$ we are given a path connected $G_{s}$-space $Z_{s}$ and a basepoint $b_{s} \in Z_{s}$ lying in some free orbit. We can find a $G_{s}$-equivariant map of pairs $f_{s}:\left(Z_{s}, G_{s} b_{s}\right) \rightarrow\left(\operatorname{Cone}\left(G_{s}\right), G_{s}\right)$. We want to define a space $\prod_{\Gamma}\left(Z_{s}, G_{s} b_{s}\right)$, together with a $G$-action on it (where $G:=\prod_{\Gamma} G_{s}$ ). It will be called the graph product of the $\left(Z_{s}, G_{s} b_{s}\right)$. The $f_{s}$ induce a map, well defined up to $G^{\prime}$-equivariant homotopy, $f: \pi_{L}\left(Z_{s}, G_{s} b_{s}\right) \rightarrow \pi_{L}\left(\operatorname{Cone}\left(G_{s}\right), G_{s}\right)$. It is easy to see that $f$ induces a surjection on fundamental groups. Pulling back the universal cover of $\pi_{L}\left(\right.$ Cone $\left.\left(G_{s}\right), G_{s}\right)$, we get a covering space, $Z \rightarrow \pi_{L}\left(Z_{s}, G_{s} b_{s}\right)$. We use the notation $\prod_{\Gamma}\left(Z_{s}, G_{s} b_{s}\right):=Z$ for this covering space. Notice that the $G^{\prime}$-action on $\pi_{L}\left(Z_{s}, G_{s} b_{s}\right)$ lifts to a $G$-action on $Z$. Also, notice that if each $Z_{s}$ is simply connected, then $Z$ is just the universal cover of the polyhedral product. Good references for graph products of groups and spaces are [24] and [27].

Example 1.5. Suppose that $\left\{\left(B_{S}, p_{s}\right)\right\}_{s \in S}$ is a collection of path connected spaces with base points and that $B=\pi_{L}\left(B_{s}, p_{s}\right)$ is the polyhedral product. For each $s \in S$, let $\pi_{s}: Z_{s} \rightarrow B_{s}$ be the universal cover and let $G_{s}=\pi_{1}\left(B_{s}, p_{s}\right)$. Let $Z^{\prime}$ denote the polyhedral product $\pi_{L}\left(Z_{s}, \pi_{s}^{-1}\left(p_{s}\right)\right)$. Then $Z^{\prime} \rightarrow B$ is a regular covering space 
with group of deck transformations $G^{\prime}$ (the product of the $G_{s}$ ). It follows that the universal cover of $Z^{\prime}$ can be identified with the graph product of the $\left(Z_{s}, \pi_{s}^{-1}\left(p_{s}\right)\right)$. Hence, $\pi_{1}(B)$ is the graph product of the $G_{s}$.

Example 1.6. Suppose that $Z_{s}=E G_{s}$, the universal cover of the classifying space $B G_{s}$. A simple argument using induction on the number of elements of $S$ (cf. [8], Remark on p. 619) shows that the polyhedral product of the $\left(B G_{s}, p_{s}\right)$ is aspherical; hence, it is a model for $B G$ and its universal cover $\prod_{\Gamma}\left(E G_{s}, G_{s} b_{s}\right)$ is $E G$.

Lemma 1.7. (i) If each $G_{s}$ acts properly on $Z_{s}$, then $G$ acts properly on the graph product $\prod_{\Gamma}\left(Z_{s}, G_{s} b_{s}\right)$.

(ii) If each $Z_{s}$ is acyclic, then so is $\prod_{\Gamma}\left(Z_{s}, G_{s} b_{s}\right)$.

Proof. The proof of (i) is trivial. For (ii), consider the cover of $Z\left(:=\prod_{\Gamma}\left(Z_{s}, G_{s} b_{s}\right)\right)$ by components of the inverse images of the $\left\{Z_{J}\right\}_{J \in \mathcal{S}(L)}$. By the Künneth Formula, each $Z_{J}$ is acyclic and the same is true for each component of its inverse image (since such a component projects homeomorphically). There is a similar cover of $\prod_{\Gamma}\left(\operatorname{Cone}\left(G_{s}\right), G_{s}\right)$ with the same nerve. So $Z$ and $\prod_{\Gamma}\left(\operatorname{Cone}\left(G_{s}\right), G_{s}\right)$ have the same homology. Since $\prod_{\Gamma}\left(\operatorname{Cone}\left(G_{s}\right), G_{s}\right)$ is the standard realization of a building, it is contractible; hence, acyclic. Statement (ii) follows.

Remark. Probably the correct level of generality at which to define the graph product of a family of pairs of spaces is the following. Suppose we are given a family of pairs $\left\{\left(Z_{s}, A_{s}\right)\right\}_{s \in S}$, where each $Z_{s}$ is path connected and where $A_{s}$ is a not necessarily connected, closed nonempty subspace. Then $\prod_{\Gamma}\left(Z_{s}, A_{s}\right)$ can be defined in the following manner. First notice that Example 1.3 works when the discrete groups $G_{s}$ are replaced by discrete spaces $D_{s}$. Let $H$ denote the fundamental group of $\pi_{L}\left(\operatorname{Cone}\left(D_{s}\right), D_{s}\right)$. If $D_{s}=\pi_{0}\left(A_{s}\right)$ denotes the set of components of $A_{s}$, then, as before, we have maps $f_{s}:\left(Z_{s}, A_{s}\right) \rightarrow\left(\operatorname{Cone}\left(D_{s}\right), D_{s}\right)$. The $f_{s}$ induce a map $f: \pi_{L}\left(Z_{s}, A_{s}\right) \rightarrow \pi_{L}\left(\operatorname{Cone}\left(D_{s}\right), D_{s}\right)$. Moreover, the induced map of fundamental groups $f_{*}: \pi_{1}\left(\pi_{L}\left(Z_{s}, A_{s}\right)\right) \rightarrow H$ is surjective. The corresponding covering space $Z$ is called the graph product of the $\left(Z_{s}, A_{s}\right)$ and is denoted by $\prod_{\Gamma}\left(Z_{s}, A_{s}\right)$. (Notice that if each $A_{s}$ is connected, then the graph product is the polyhedral product, $\pi_{L}\left(Z_{s}, A_{s}\right)$.) In particular, this allows us to deal with the case where each $A_{s}$ is a $G_{s}$-orbit (not necessarily a free $G_{s}$-orbit). So suppose that $A_{s}=G_{s} / H_{s}$. Then the group of lifts of the $G^{\prime}$-action to the universal cover of $\pi_{L}\left(\operatorname{Cone}\left(G_{s} / H_{S}\right), G_{s} / H_{s}\right)$ is the "graph product of pairs", $\prod_{\Gamma}\left(G_{s}, H_{s}\right)$, defined previously in [27].

\section{A spectral sequence}

A poset of coefficients is a contravariant functor $\mathcal{A}$ from a poset $\mathcal{P}$ to the category of abelian groups (i.e., it is a collection $\left\{\mathcal{A}_{a}\right\}_{a \in \mathcal{P}}$ of abelian groups together with 
homomorphisms $\varphi_{b a}: \mathcal{A}_{a} \rightarrow \mathcal{A}_{b}$, defined whenever $a>b$, such that $\varphi_{c a}=\varphi_{c b} \varphi_{b a}$, whenever $a>b>c$ ). The functor $\mathcal{A}$ also gives us a system of coefficients on the cell complex $\operatorname{Flag}(\mathcal{P})$ : it associates to the simplex $\sigma$ the abelian group $\mathcal{A}_{\min (\sigma)}$. Hence, we get a cochain complex

$$
C^{j}(\operatorname{Flag}(\mathcal{P}) ; \mathcal{A}):=\bigoplus_{\sigma \in \operatorname{Flag}(\mathcal{P})^{(j)}} \mathcal{A}_{\min (\sigma)}
$$

where $\operatorname{Flag}(\mathcal{P})^{(j)}$ means the set of $j$-simplices in $\operatorname{Flag}(\mathcal{P})$.

Given a CW complex $Y$, a poset of spaces in $Y$ over $\mathcal{P}$ is a cover $\mathcal{V}=\left\{Y_{a}\right\}_{a \in \mathcal{P}}$ of $Y$ by subcomplexes indexed by $\mathcal{P}$ so that if $N(\mathcal{V})$ denotes the nerve of the cover, then

(i) $a<b \Longrightarrow Y_{a} \subset Y_{b}$, and

(ii) the vertex set $\operatorname{Vert}(\sigma)$ of each simplex of $N(\mathcal{V})$ has the greatest lower bound $\wedge \sigma$ in $\mathcal{P}$, and

(iii) $\mathcal{V}$ is closed under taking finite nonempty intersections, i.e., for any simplex $\sigma$ of $N(\mathcal{V})$,

$$
\bigcap_{a \in \sigma} Y_{a}=Y_{\wedge \sigma}
$$

Remark. Any cover leads to a poset of spaces by taking all nonempty intersections as elements of the new cover, and removing duplicates. The resulting poset is the set of all nonempty intersections, ordered by inclusion.

Lemma 2.1 (cf. [23]). Suppose that $\mathcal{V}=\left\{Y_{a}\right\}_{a \in \mathcal{P}}$ is a poset of spaces for $Y$ over $\mathcal{P}$. There is a Mayer-Vietoris type spectral sequence converging to $H^{*}(Y)$ with $E_{1}$-term

$$
E_{1}^{i, j}=C^{i}\left(\operatorname{Flag}(\mathcal{P}) ; \mathscr{H}^{j}(\mathcal{V})\right),
$$

and $E_{2}$-term

$$
E_{2}^{i, j}=H^{i}\left(\operatorname{Flag}(\mathcal{P}) ; \mathscr{H}^{j}(\mathcal{V})\right),
$$

where the coefficient system $\mathscr{H}^{j}(\mathcal{V})$ is given by $\mathscr{H}^{j}(\mathcal{V})(\sigma)=H^{j}\left(Y_{\min (\sigma)}\right)$.

Proof. We follow the line laid down in [6], Chapter VII, §3, 4. Consider the double complex

$$
E_{0}^{i, j}=\bigoplus_{\substack{\sigma \in \operatorname{Flag}(\mathcal{P}) \\ \operatorname{dim} \sigma=i}} C^{j}\left(Y_{\min (\sigma)}\right),
$$

where the differentials are defined as follows. The vertical differentials are direct sums of the differentials $d: C^{j}\left(Y_{\min (\sigma)}\right) \rightarrow C^{j+1}\left(Y_{\min (\sigma)}\right)$. Similarly, the horizontal differentials are direct sums of homomorphisms $\delta: C^{j}\left(Y_{\min (\sigma)}\right) \rightarrow C^{j}\left(Y_{\min (\tau)}\right)$ where the matrix entry corresponding to $\sigma \tau$ is $[\tau: \sigma] i_{\tau \sigma}$, where $[\tau: \sigma]$ is the incidence number and $i_{\tau \sigma}: C^{j}\left(Y_{\min (\sigma)}\right) \rightarrow C^{j}\left(Y_{\min (\tau)}\right)$ is the restriction map if $\tau$ is a coface of $\sigma$ and 0 otherwise. As in [6], p. 165, there are two spectral sequences 
associated to the double complex. The first begins by taking vertical cohomology to get $E_{1}$ and then takes horizontal cohomology to get $E_{2}$. The differential on the $E_{r}$ sheet has bidegree $(r,-r+1)$. The second spectral sequence begins with the horizontal differential so that the differential on the $E_{r}$ sheet has bidegree $(-r+1, r)$

The usual inclusion-exclusion argument using properties (i)-(iii) of a poset of spaces shows that the rows of the double complex are exact, except when $i=0$, where we get $C^{j}(Y)$ as the $E_{1}^{0, j}$-term of the second spectral sequence. This implies that the second spectral sequence collapses at $E_{2}$ and that the cohomology of the double complex is $H^{*}(Y)$ (cf. the exercise in [6], p. 165).

We can rewrite (2.1) as $E_{0}^{i, j}=C^{i}\left(\operatorname{Flag}(\mathcal{P}) ; \mathcal{C}^{j}(\mathcal{V})\right)$, where the coefficient systems are defined by $\mathcal{C}^{j}(\mathcal{V})(\sigma)=C^{j}\left(Y_{\min (\sigma)}\right)$. So the first spectral sequence is the one claimed in the lemma.

For $a \in \mathcal{P}$, let $Y_{<a}:=\bigcup_{b<a} Y_{b}$. For any maximal element $a \in \mathcal{P}$, put $Y_{\neq a}:=$ $\bigcup_{b \neq a} Y_{b}$. Consider the following two conditions on the poset of spaces.

(Z') For any $a, b \in \mathcal{P}$ with $b<a$, the induced homomorphism $H^{*}\left(Y_{a}\right) \rightarrow H^{*}\left(Y_{b}\right)$ is the zero map.

(Z) For any $a \in \mathcal{P}$, the induced homomorphism $H^{*}\left(Y_{a}\right) \rightarrow H^{*}\left(Y_{<a}\right)$ is the zero map.

Note that (Z) implies ( $\left.\mathrm{Z}^{\prime}\right)$ since the map $H^{*}\left(Y_{a}\right) \rightarrow H^{*}\left(Y_{b}\right)$ factors through $H^{*}\left(Y_{<a}\right)$.

Lemma 2.2. Suppose that $\mathcal{V}=\left\{Y_{a}\right\}_{a \in \mathcal{P}}$ is a poset of spaces for $Y$ over $\mathcal{P}$.

(i) If ( $\left.\mathrm{Z}^{\prime}\right)$ holds, then

$$
E_{2}^{i, j}=\bigoplus_{a \in \mathcal{P}} H^{i}\left(\operatorname{Flag}\left(\mathcal{P}_{\geq a}\right), \operatorname{Flag}\left(\mathcal{P}_{>a}\right) ; H^{j}\left(Y_{a}\right)\right) .
$$

(ii) If (Z) holds, then the spectral sequence degenerates at $E_{2}$ and

$$
\operatorname{Gr} H^{*}(Y)=\bigoplus_{a \in \mathcal{P}} H^{i}\left(\operatorname{Flag}\left(\mathcal{P}_{\geq a}\right), \operatorname{Flag}\left(\mathcal{P}_{>a}\right) ; H^{j}\left(Y_{a}\right)\right) .
$$

Proof. We use the double complex from the proof of Lemma 2.1. The cochain group decomposes as a direct sum:

$$
C^{i}\left(\operatorname{Flag}(\mathcal{P}) ; \mathcal{C}^{j}(\mathcal{V})\right)=\bigoplus_{a \in \mathcal{P}} C^{i}\left(\operatorname{Flag}\left(\mathcal{P}_{\geq a}\right), \operatorname{Flag}\left(\mathcal{P}_{>a}\right) ; C^{j}\left(Y_{a}\right)\right) .
$$

The vertical differentials at $E_{0}$ respect this decomposition, so at $E_{1}$ the spectral sequence always decomposes as a direct sum:

$$
E_{1}^{i, j}=\bigoplus_{a \in \mathcal{P}} C^{i}\left(\operatorname{Flag}\left(\mathcal{P}_{\geq a}\right), \operatorname{Flag}\left(\mathcal{P}_{>a}\right) ; H^{j}\left(Y_{a}\right)\right) .
$$

In general, the differentials at $E_{1}$ do not respect this decomposition; however, condition $\left(Z^{\prime}\right)$ implies that they do, and therefore, the spectral sequence also decomposes 
at $E_{2}$ :

$$
E_{2}^{i, j}=\bigoplus_{a \in \mathcal{P}} H^{i}\left(\operatorname{Flag}\left(\mathcal{P}_{\geq a}\right), \operatorname{Flag}\left(\mathcal{P}_{>a}\right) ; H^{j}\left(Y_{a}\right)\right)
$$

This proves (i).

Now suppose that $(Z)$ holds. By induction, we can assume that (ii) is true for all posets with fewer elements. If $z \in E_{0}^{i, j}$ is a vertical cocycle, then its higher differential is given by $d_{r}(z)=\delta\left(x_{r}\right)$, where $\left(x_{0}=z, x_{1}, \ldots, x_{r}\right)$ is any sequence of elements satisfying $x_{k} \in E_{0}^{i+k, j-k}$ and $\delta\left(x_{k}\right)=d\left(x_{k+1}\right)$. Since the columns of double complex split as direct sums over $\sigma$, the vertical cocycles split as a direct sum, and it suffices to show that higher differentials vanish for each summand. So let $\sigma$ be a simplex in $\operatorname{Flag}(\mathcal{P})$, and consider the term $C^{j}\left(Y_{\min (\sigma)}\right)$. There are two cases.

1) $\sigma$ is a face of a simplex $\tau$ with $\min (\tau)=\min (\sigma)$. Then $i_{\tau \sigma}$ is the identity map and this forces higher differentials to be trivial on this term. Indeed, if $z \in$ $C^{j}\left(Y_{\min (\sigma)}\right)$, then $d\left(x_{1}\right)=\delta(z)$, and therefore $d\left(x_{1 \tau}\right)= \pm z$, where $x_{1 \tau}$ denotes the $\tau$ component of $x_{1}$. Thus we can choose $x_{1}^{\prime}= \pm \delta\left(i_{\tau \sigma}^{-1}\left(x_{1 \tau}\right)\right)$ and all higher $x_{k}=0$.

2) $\sigma$ is a "maximal" face, i.e., all its cofaces have strictly smaller minimum vertices. Then $a=\max (\sigma)$ is a maximal element of $\mathcal{P}$. The cover of $Y_{\neq a}$ by $\left\{Y_{b} \mid b \neq a\right\}$ is a poset of spaces over $\mathcal{P}_{\neq a}$. Let $E_{0, a}$ be the subcomplex of $E_{0}$ corresponding to the pair $\left(Y, Y_{\neq a}\right)$ :

$$
E_{0, a}^{i, j}=\bigoplus_{\substack{\max (\tau)=a \\ \operatorname{dim} \tau=i}} C^{j}\left(Y_{\min (\tau)}\right) .
$$

Note that $C^{j}\left(Y_{\sigma}\right)$ is contained in this subcomplex, so it suffices to show that the higher differentials vanish for $E_{0, a}$. The pair $\left(Y, Y_{\neq a}\right)$ excises to the pair $\left(Y_{a}, Y_{<a}\right)$. So we have a short exact sequence

$$
0 \rightarrow E_{0, a} \rightarrow E_{0, \leq a} \rightarrow E_{0,<a} \rightarrow 0
$$

where

$$
E_{0, \leq a}^{i, j}=\bigoplus_{\substack{\tau \in \mathrm{Flag}(\mathcal{P} \leq a) \\ \operatorname{dim} \tau=i}} C^{j}\left(Y_{\min (\tau)}\right)
$$

and

$$
E_{0,<a}^{i, j}=\bigoplus_{\substack{\tau \in \operatorname{Flag}(\mathcal{P}<a) \\ \operatorname{dim} \tau=i}} C^{j}\left(Y_{\min (\tau)}\right) .
$$

are double complexes corresponding to the covers (posets of spaces) of $Y_{a}$ by $\left\{Y_{b} \mid\right.$ $b \leq a\}$ and of $Y_{<a}$ by $\left\{Y_{b} \mid b<a\right\}$.

The $E_{2}$-terms of the spectral sequences $E_{\leq a}$ and $E_{<a}$ are

$$
E_{2, \leq a}^{i, j}=\bigoplus_{b \leq a} H^{i}\left(\operatorname{Flag}(\mathcal{P}[b, a]), \operatorname{Flag}(\mathcal{P}(b, a]) ; H^{j}\left(Y_{b}\right)\right),
$$

and

$$
E_{2,<a}^{i, j}=\bigoplus_{b<a} H^{i}\left(\operatorname{Flag}(\mathcal{P}[b, a)), \operatorname{Flag}(\mathcal{P}(b, a)) ; H^{j}\left(Y_{b}\right)\right)
$$


For $b<a$, $\operatorname{Flag}([b, a])$ is a cone on $\operatorname{Flag}((b, a])$; therefore, the only nonzero terms in $E_{2, \leq a}$ come from $b=a$ and $i=0$, i.e., $E_{2, \leq a}$ has $H^{j}(Y)$ in the 0 -row and 0 everywhere else. In particular, it collapses at $E_{2}$. By inductive assumption $E_{2,<a}$ also collapses at $E_{2}$. Since, by hypothesis, $H^{*}\left(Y_{a}\right) \rightarrow H^{*}\left(Y_{<a}\right)$ is the 0-map, the long exact sequence of the pair $\left(Y_{a}, Y_{<a}\right)$ splits into short exact sequences, and similarly for the $E_{2}$-terms, it follows that the spectral sequence $E_{a}$ also collapses at $E_{2}$-term. Thus, the higher differentials in $E$ are 0 .

\section{Some previous cohomology computations}

Suppose that $G$ is a discrete group and $Y$ is a $G$-CW complex. Let $N$ be a left $G$-module. The $G$-equivariant cochain complex is defined by,

$$
C_{G}^{i}(Y ; N):=\operatorname{Hom}_{G}\left(C_{i}(Y) ; N\right),
$$

where $C_{*}(Y)$ denotes the usual cellular chain complex. Its cohomology is denoted $H_{G}^{*}(Y ; N)$. If $G$ acts freely on $Y$, then $C_{G}^{*}(Y ; N)$ can be identified with $C^{*}(Y / G ; N)$, the cellular cochains on the quotient space with local coefficients in $N$. There is a similar result even when the action is not free; however, the coefficients will no longer be locally constant. Rather, the coefficients will be in a contravariant functor $\mathcal{I}(N)$ from the poset of cells in $Y / G$ to the category of abelian groups: $\mathcal{I}(N)$ assigns to a cell $c$ the fixed submodule $N^{G_{c}}$, where $G_{c}$ denotes the stabilizer of some lift of $c$ and where

$$
C_{G}^{i}(Y)=C^{i}(Y / G ; \mathcal{I}(N)) .
$$

For $Y=E G$, the universal cover of the classifying space $B G$, define the cochains and cohomology of $G$ with coefficients in $N$ by

$$
\begin{aligned}
& C^{*}(G ; N):=C_{G}^{*}(E G ; N)=C^{*}(B G ; N), \\
& H^{*}(G ; N):=H_{G}^{*}(E G ; N)=H^{*}(B G ; N) .
\end{aligned}
$$

The spectral sequence arguments from $\S 2$ work with equivariant cochains (in particular with cochains with local coefficients) as long as the cover $\mathcal{V}$ is $G$-equivariant.

In what follows we will be interested principally in two cases: $N=\mathbb{Z} G$, the group ring, and $N=\mathcal{N}(G)$, the group von Neumann algebra. We recall the definitions and some results which have been proved previously.

Group ring coefficients. In the case of group ring coefficients, if $G$ acts properly and cocompactly on $Y$, there is the following formula (cf. [6], Example 4, p. 209),

$$
H_{G}^{*}(Y ; \mathbb{Z} G)=H_{c}^{*}(Y),
$$

where $H_{c}^{*}(Y)$ means cohomology with compact supports. Thus, Lemma 1.7 implies the following. 
Corollary 3.1. For each $s \in S$, suppose that $G_{s}$ is a discrete group and that $\left(Z_{s}, G_{s} b_{s}\right) a G_{s}-C W$ complex together with a free orbit. Also suppose that each $G_{s}$-action is proper and cocompact and that $Z_{s}$ is acyclic. Then for $G=\prod_{\Gamma} G_{s}$ and $Z=\prod_{\Gamma}\left(Z_{s}, G_{s} b_{s}\right)$, we have

$$
H^{*}(G ; \mathbb{Z} G)=H_{c}^{*}(Z)
$$

The cohomology groups of Coxeter groups are computed as follows.

Theorem 3.2 ([10] as well as [14]).

$$
H^{n}(W ; \mathbb{Z} W)=H_{c}^{n}(U(W, K))=\underset{J \in S(W, S)}{\bigoplus} H^{n}\left(K, K^{S-J}\right) \otimes \widehat{A}(W)^{J},
$$

where $\hat{A}(W)^{J}$ is the free abelian group on the set of $w \in W$ which have reduced expressions ending with letters in $J$.

Using this, Jensen and Meier proved the following. (A different proof of this will be given in §4.1.)

Theorem 3.3 (Jensen-Meier [28]). Suppose that $(W, S)$ is a RACS and $A_{L}$ is the associated RAAG. Then

$$
H^{n}\left(A_{L} ; \mathbb{Z} A_{L}\right)=\bigoplus_{J \in S(W, S)} H^{n-|J|}\left(K_{J}, \partial K_{J}\right) \otimes \mathbb{Z}\left(A_{L} / A_{J}\right) .
$$

Theorem 3.4 ([13], Corollary 8.2). Suppose that $\mathcal{C}$ is a locally finite building of type $(W, S)$. Then

$$
H_{c}^{n}(\mathcal{B}(\mathcal{C}, K))=\bigoplus_{J \in S(W, S)} H^{n}\left(K, K^{S-J}\right) \otimes \widehat{A}(\mathcal{C})^{J},
$$

where $\hat{A}(\mathcal{C})^{J}$ is a certain subgroup of the free abelian group on $\mathcal{C}$.

In particular, by Lemma 1.1, this theorem gives the following computation of [14] of the compactly supported cohomology of a locally finite RAB and hence, of the cohomology with group ring coefficients of the graph product of a collection of finite groups.

Theorem 3.5 ([14], Theorem 6.6). Suppose that $\left\{G_{s}\right\}_{s \in S}$ is a collection of finite groups and that $G=\prod_{\Gamma} G_{s}$. Then

$$
H^{n}(G ; \mathbb{Z} G)=\bigoplus_{J \in S(L)} H^{n}\left(K, K^{S-J}\right) \otimes \widehat{A}(J),
$$

where $\widehat{A}(J)$ is a certain (free abelian) subgroup of $\mathbb{Z}\left(G / G_{J}\right)$. 
$L^{2}$-cohomology and $L^{2}$-Betti numbers. The real group algebra, $\mathbb{R} G$, of $G$ consists of all finitely supported functions $G \rightarrow \mathbb{R}$. Its standard basis is $\left\{e_{g}\right\}_{g \in G}$, where $e_{g}$ denotes the indicator function of $\{g\}$. The standard inner product on $\mathbb{R} G$ is defined by $e_{g} \cdot e_{h}=\delta_{g h}$, where $\delta_{g h}$ is the Kronecker delta. The Hilbert space completion of $\mathbb{R} G$, denoted $L^{2}(G)$, consists of all square summable functions $G \rightarrow \mathbb{R}$. The group $G$ acts orthogonally on $L^{2}(G)$ by either left or right translation. To fix ideas, let us say that it is the right action defined by left translation. The von Neumann algebra of $G$, denoted by $\mathcal{N}(G)$, is the commutant of the $G$-action. It acts on $L^{2}(G)$ from the left. For $\varphi \in \mathcal{N}(G)$, define

$$
\operatorname{tr}_{\mathcal{N}(G)}(\varphi):=\left(\varphi e_{1}\right) \cdot\left(e_{1}\right) .
$$

If $V$ is a closed $G$-stable subspace of a finite direct sum of copies of $L^{2}(G)$, then its von Neumann dimension is defined by

$$
\operatorname{dim}_{\mathcal{N}(G)} V:=\operatorname{tr}_{\mathcal{N}(G)}\left(p_{V}\right),
$$

where $p_{V}: \bigoplus L^{2}(G) \rightarrow \bigoplus L^{2}(G)$ is orthogonal projection onto $V$.

Suppose that the $G$-CW complex $Y$ is proper and cocompact. Define $L^{2} C^{*}(Y)$ to be the cochain complex of real-valued, square summable cochains on $Y$. Denote its reduced cohomology groups by $L^{2} H^{*}(Y)$. (Here "reduced" means $\operatorname{Ker} \delta / \overline{\operatorname{Im} \delta}$, where $\delta: L^{2} C^{i}(Y) \rightarrow L^{2} C^{i+1}(Y)$ is the coboundary operator. It is necessary to take the closure of $\operatorname{Im} \delta$ for the quotient to be a Hilbert space.) Define the $i^{t \text { th }} L^{2}$-Betti number by

$$
L^{2} b^{i}(Y ; G):=\operatorname{dim}_{\mathcal{N}(G)} L^{2} H^{i}(Y) .
$$

If $Y$ is acyclic, then $L^{2} H^{i}(Y)$ depends only on $G$ and is denoted by $L^{2} H^{i}(G)$ and similarly, $L^{2} b^{i}(G):=L^{2} b^{i}(Y ; G)$. Thus, Lemma 1.7 implies the following.

Corollary 3.6. For each $s \in S$, suppose that $G_{s}$ is a discrete group and that $\left(Z_{s}, G_{s} b_{s}\right)$ is a $G_{s}-C W$ complex together with a free orbit. Also suppose that each $G_{s}$-action is proper and cocompact and that $Z_{s}$ is acyclic. Then for $G=\prod_{\Gamma} G_{s}$ and $Z=\prod_{\Gamma}\left(Z_{s}, G_{s} b_{s}\right)$, we have

$$
L^{2} b^{*}(G)=L^{2} b^{*}(Z ; G) .
$$

The $L^{2}$-Betti numbers of Coxeter groups have proved to be difficult to compute. Some partial results and conjectures can be found in [19]. For locally finite buildings of very large thickness there is a complete calculation due to Dymara-Januszkiewicz [22]. The requirement on the thickness is reduced in [15] (cf. Theorems 6.3 and 6.4 in $\$ 6.2$ ).

In the case of Artin groups, we have the following easy computation of [17]. (A proof of this will be given in the next section.)

Theorem 3.7 ([17]). $L^{2} b^{n}(\tilde{X} ; A)=b^{n}(K, \partial K)=\bar{b}^{n-1}(L)$, where, as usual, $b^{n}(K, \partial K)=\operatorname{dim}\left(H^{n}(K, \partial K ; \mathbb{R})\right)$ and $\bar{b}^{n-1}(L)=\operatorname{dim}\left(\bar{H}^{n-1}(L ; \mathbb{R})\right)$. 
In [30], Lück shows that there is an equivalence of categories between the category of finitely generated $\mathcal{N}(G)$-modules and the category of orthogonal representations of $G$ on Hilbert spaces which are $G$-isomorphic to closed, $G$-stable subspaces of a finite direct sum of copies of $L^{2}(G)$. Given a finitely generated $\mathcal{N}(G)$-module $E$, define $\operatorname{dim}_{\mathcal{N}(G)} E$ to be the von Neumann dimension of the corresponding Hilbert space. Then

$$
L^{2} b^{i}(Y ; G)=\operatorname{dim}_{\mathcal{N}(G)} H_{G}^{i}(Y ; \mathcal{N}(G)) .
$$

Just as in (3.1), we have that

$$
H_{G}^{*}(Y ; \mathcal{N}(G))=H^{*}(Y / G ; \mathcal{I}(\mathcal{N}(G))) .
$$

\section{New computations}

4.1. Artin groups. As in $\S 1.3, A$ is the Artin group associated to a Coxeter system $(W, S)$ and $X$ is its Salvetti complex. As usual, $L=L(W, S), \delta=\delta(W, S)$ and $K:=\operatorname{Flag}(S)$. We wish to compute $H^{*}(X ; \mathbb{Z} A)$. Given a spherical subset $J \in S$, $A_{J}$ is the corresponding Artin group and $X_{J}$ is its Salvetti complex. We know that $X_{J}$ is the classifying space for $A_{J}$. By [31] (see also [3]), for each spherical subset $J, A_{J}$ is a duality group of dimension $|J|$. This means that $H^{*}\left(A_{J} ; \mathbb{Z} A_{J}\right)$ is zero for $* \neq|J|$ and that $F_{J}:=H^{|J|}\left(A_{J} ; \mathbb{Z} A_{J}\right)$ is free abelian.

As explained in $\S 1.3$, the cover $\mathcal{V}=\left\{X_{J}\right\}_{J \in S}$ is a poset of spaces for $X$. In the case of group ring coefficients, we have a spectral sequence of the type considered in $\$ 2$ converging to $H^{*}(X ; \mathbb{Z} A)$. It has $E_{2}$-term: $E_{2}^{i, j}=H^{i}\left(K ; \mathscr{H}^{j}(\mathcal{V})\right)$, where $\mathscr{H}^{j}(\mathcal{V})$ is the coefficient system, $\sigma \mapsto H^{i}\left(X_{\min \sigma} ; \mathbb{Z} A\right)$,. By Lemma 2.2, once we establish condition $(Z)$ of $\S 2$ we will get the following calculation.

\section{Theorem 4.1.}

$$
\operatorname{Gr} H^{n}(X ; \mathbb{Z} A)=\bigoplus_{J \in \mathcal{S}(W, S)} H^{n-|J|}\left(K_{J}, \partial K_{J}\right) \otimes H^{|J|}\left(A_{J} ; \mathbb{Z} A\right) .
$$

A similar argument can be used recover the calculation of the $L^{2}$-Betti numbers of $X$ in [17]. (This computation was stated earlier as Theorem 3.7.) The spectral sequence has $E_{2}^{i, j}=H^{i}\left(K ; \mathscr{H}^{j}(\mathcal{V})\right)$, where $\mathscr{H}^{j}(\mathcal{V})$ is the coefficient system $\sigma \mapsto$ $H^{i}\left(X_{\min \sigma} ; \mathcal{N}(A)\right)$. The key observation in [17] for proving Theorem 3.7 was that for $J \neq \emptyset$, all $L^{2}$-Betti numbers of $A_{J}$ vanish. In particular, condition (Z) of $\S 2$ holds (since all cohomology groups vanish except when $J=\emptyset$ ). So $E_{1}^{i, j}$ is 0 for $j \neq 0$ while

$$
E_{1}^{i, 0}=C^{i}(K, \partial K ; \mathcal{N}(A)),
$$

where the coefficients are now constant. Therefore $H^{n}(X ; \mathcal{N}(A)) \cong H^{n}(K, \partial K) \otimes$ $\mathcal{N}(A)$; whence, Theorem 3.7.

In the case of Theorem 4.1, condition (Z) is basically the following lemma. 
Lemma 4.2. For any $J \in S, H^{*}\left(X_{J} ; \mathbb{Z} A\right)$ is concentrated in degree $|J|$, where it is equal to the free abelian group $F_{J} \otimes_{A_{J}} \mathbb{Z} A$. Hence, $H^{*}\left(X_{J} ; \mathbb{Z} A\right) \rightarrow H^{*}\left(X_{<J} ; \mathbb{Z} A\right)$ is the zero map.

Proof. The first sentence is from [31]. The second sentence follows since $X_{J}$ is a $|J|$-dimensional $\mathrm{CW}$ complex, $H^{*}\left(X_{J} ; \mathbb{Z} A_{J}\right)$ is concentrated in the top dimension and $X_{<J}$ is a subcomplex of one less dimension.

Theorem 4.1 follows immediately from Lemma 2.2. We note that if the $K(\pi, 1)$ Conjecture holds for $A$ (i.e, if $X=B A$ ), then the formula in Theorem 4.1 is a calculation of $H^{*}(A ; \mathbb{Z} A)$ and Theorem 3.7 gives a formula for $L^{2} b^{n}(A)$. In particular, since the $K(\pi, 1)$-Conjecture holds for RAAG's, Theorem 4.1 gives as a corollary, a different proof the Jensen-Meier calculation in [28] (stated previously as Theorem 3.3).

4.2. Bestvina-Brady groups. In this section $A_{L}$ is a RAAG, $T_{L}$ is its Salvetti complex defined in (1.2) and $\pi: A_{L} \rightarrow \mathbb{Z}$ is the standard homomorphism. Its kernel $\mathrm{BB}_{L}$ is the Bestvina-Brady group. We have a $\pi$-equivariant map $p: \widetilde{T}_{L} \rightarrow \mathbb{R}$. Put $Z_{L}=p^{-1}(t)$ for some $t \in \mathbb{R}-\mathbb{Z}$ (say for $t=\frac{1}{2}$ ). Then $\mathrm{BB}_{L}$ acts on $Z_{L}$. It is proved in [4] that if $L$ is acyclic, then so is $Z_{L}$ and hence, $\mathrm{BB}_{L}$ is type FP. We can compute equivariant cohomology groups of $Z_{L}$ by the method used in the proof of Theorem. 4.1.

Theorem 4.3. The compactly supported cohomology of $Z_{L}$ is given by

$$
\begin{aligned}
H_{c}^{n}\left(Z_{L}\right) & =H_{\mathrm{BB}_{L}}^{n}\left(Z_{L} ; \mathbb{Z} \mathrm{BB}_{L}\right) \\
& =\bigoplus_{J \in \mathcal{S}(L)_{>}} H^{n-|J|+1}\left(K_{J}, \partial K_{J}\right) \otimes \mathbb{Z}\left(\mathrm{BB}_{L} /\left(\mathrm{BB}_{L} \cap A_{J}\right)\right) .
\end{aligned}
$$

When $L$ is acyclic, this is isomorphic to the compactly supported cohomology of $\widetilde{T}_{L}$ shifted down in degree by 1 ; moreover, it gives a calculation of $H^{n}\left(\mathrm{BB}_{L} ; \mathbb{Z} \mathrm{BB}_{L}\right)$.

Remark. If $L$ is $R$-acyclic for some commutative ring $R$, then the same formula gives a calculation of $H^{n}\left(\mathrm{BB}_{L} ; R \mathrm{BB}_{L}\right)$.

Proof. We intersect the cover $\left\{\widetilde{T}_{J}\right\}_{J \in S(L)}$ of $\widetilde{T}_{L}$ with $Z_{L}$. Put $Z_{J}:=Z_{L} \cap \widetilde{T}_{J}$. Since $t$ is not an integer, $Z_{L}$ does not contain any vertices of the cubical complex $\widetilde{T}_{L}$, i.e., $Z_{L} \cap \widetilde{T}^{\emptyset}=\emptyset$. On the other hand, when $J$ is nonempty, the intersection of $Z_{L}$ with any component of $\widetilde{T}_{J}$ is a Euclidean subspace of codimension one and the collection of such intersections is in one-to-one correspondence with the cosets of $\mathrm{BB}_{L} \cap A_{J}$ in $\mathrm{BB}_{L}$. So $\left\{Z_{J}\right\}_{J \in S(L)_{>\emptyset}}$ is a poset of spaces on $Z_{L}$. The simplicial complex $\operatorname{Flag}\left(S(L)_{>\emptyset}\right)$ is equal to $\partial K$ (i.e., the barycentric subdivision of $L$ ). The $E_{1}^{i, j}$-term of the spectral sequence is $C^{i}\left(K ; \mathscr{H}^{j}(\mathcal{V})\right)$, where the coefficient system takes $\sigma$ to $H_{\mathrm{BB}_{L}}^{j}\left(Z_{\min \sigma} ; \mathbb{Z} \mathrm{BB}_{L}\right)$. Since each component of $Z_{J}$ is Euclidean space of 
dimension $|J|-1$, the coefficients, $H_{\mathrm{BB}_{L}}^{j}\left(Z_{J} ; \mathbb{Z B B}_{L}\right)$ are 0 whenever $j \neq|J|-1$. Moreover, for $j=|J|-1$,

$$
H_{\mathrm{BB}_{L}}^{j}\left(Z_{J} ; \mathbb{Z B B} B_{L}\right)=H_{c}^{j}\left(\mathbb{R}^{j}\right) \otimes_{\mathrm{BB}_{L} \cap A_{J}} \mathbb{Z} \mathrm{BB}_{L}=\mathbb{Z}\left(\mathrm{BB}_{L} /\left(\mathrm{BB}_{L} \cap A_{J}\right)\right) .
$$

It follows that conditions $\left(Z^{\prime}\right)$ and $(Z)$ of $\S 2$ hold (because $Z_{<J}$ is a subcomplex of dimension $|J|-2$ ). Hence, by Lemma 2.2, the spectral sequence degenerates at $E_{2}$ and $E_{2}^{i, j}=\bigoplus_{J} E_{2, J}^{i, j}$, where $E_{2, J}^{i, j}$ is nonzero only for $j=|J|-1$, in which case,

$$
E_{2, J}^{i,|J|-1}=H^{i}\left(K_{J}, \partial K_{J}\right) \otimes \mathbb{Z}\left(\mathrm{BB}_{L} /\left(\mathrm{BB}_{L} \cap A_{J}\right)\right) .
$$

The theorem follows. (We also note that for $J \neq \emptyset, \pi: A_{J}=\mathbb{Z}^{J} \rightarrow \mathbb{Z}$ is onto, so that $\mathrm{BB}_{L} /\left(\mathrm{BB}_{L} \cap A_{J}\right) \cong A_{L} / A_{J}$.

Similarly, we compute the $L^{2}$-Betti numbers of $Z_{L}$ as follows.

Theorem 4.4. Suppose that $Z_{L}$ is a generic level set of the function $p: \widetilde{T}_{L} \rightarrow \mathbb{R}$. Then

$$
L^{2} b^{n}\left(Z_{L} ; \mathrm{BB}_{L}\right)=\sum_{s \in S} b^{n}\left(K_{s}, \partial K_{s}\right)=\sum_{s \in S} \bar{b}^{n-1}(\operatorname{Lk}(s)) .
$$

In particular, when $L$ is $\mathbb{Q}$-acyclic,

$$
L^{2} b^{n}\left(\mathrm{BB}_{L}\right)=\sum_{s \in S} b^{n}\left(K_{s}, \partial K_{s}\right) .
$$

Proof. As before, the $E_{1}^{i, j}$-term of the spectral sequence is $C^{i}\left(K ; \mathscr{H}^{j}(\mathcal{V})\right)$, where the coefficient system takes $\sigma$ to $H^{j}\left(Z_{\min \sigma} ; \mathcal{N}\left(\mathrm{BB}_{L}\right)\right)$. Since each component of $Z_{J}$ is Euclidean space of dimension $|J|-1$, the coefficients, $H^{*}\left(Z_{J} ; \mathcal{N}\left(\mathrm{BB}_{L}\right)\right)$ are 0 whenever $|J| \neq 1$. Hence, by Lemma 2.2, the spectral sequence degenerates at $E_{2}$ and only $E_{2}^{i, 0}$ can be nonzero, where

$$
E_{2}^{i, 0}=\bigoplus_{s \in S} H^{i}\left(K_{s}, \partial K_{s} ; \mathcal{N}\left(\mathrm{BB}_{L}\right)\right)
$$

The theorem follows.

Remark. Here is a different proof of Theorem 4.3 when $L$ is acyclic. Put $Y_{+}:=$ $p^{-1}\left(\left[\frac{1}{2}, \infty\right)\right)$ and $Y_{-}:=p^{-1}\left(\left(\infty, \frac{1}{2}\right]\right)$. We first claim that the compactly supported cohomology of $Y_{ \pm}$vanishes in all degrees. It suffices to consider $Y_{+}$, the argument for $Y_{-}$being similar. The arguments of [4] show that when $L$ is acyclic the inclusion of any level set $p^{-1}(t)$ into a sublevel set $p^{-1}\left(\left[\frac{1}{2}, t\right]\right)$ induces an isomorphism on homology. The same argument shows that it induces an isomorphism on compactly supported cohomology, $H_{c}^{*}\left(p^{-1}\left(\left[\frac{1}{2}, t\right]\right)\right) \rightarrow H_{c}^{*}\left(p^{-1}(t)\right)$. Hence, $H_{c}^{*}\left(p^{-1}\left(\left[\frac{1}{2}, t\right]\right), p^{-1}(t)\right)=0$. Since there is an excision, $H_{c}^{*}\left(Y_{+}, p^{-1}([t, \infty))\right) \cong$ 
$H_{c}^{*}\left(p^{-1}\left(\left[\frac{1}{2}, t\right]\right), p^{-1}(t)\right)$, the left-hand side also vanishes. For any compact subset $C \subset Y_{+}$we have that $Y_{+}-C \supset p^{-1}([t, \infty])$ for large enough $t$; so

$$
H_{c}^{*}\left(Y_{+}\right)=\lim _{t \rightarrow \infty} H_{c}^{*}\left(Y_{+}, p^{-1}([t, \infty))\right),
$$

and by the previous discussion the right-hand side vanishes. Hence, so does $H_{c}^{*}\left(Y_{+}\right)$. We have $Y_{+} \cup Y_{-}=\widetilde{T}_{L}$ and $Y_{+} \cap Y_{-}=Z_{L}$ and a Mayer-Vietoris sequence

$$
0=H_{c}^{*}\left(Y_{+}\right) \oplus H_{c}^{*}\left(Y_{-}\right) \rightarrow H_{c}^{*}\left(Z_{L}\right) \rightarrow H_{c}^{*+1}\left(\widetilde{T}_{L}\right) \rightarrow 0 .
$$

The theorem follows from the computation of $H_{c}^{*+1}\left(\widetilde{T}_{L}\right)$ in Theorem 4.1 (or in Theorem 3.3).

Remark. Theorem 3.3 provides a calculation of $H^{*}\left(A_{L} ; \mathbb{Z} A_{L}\right)$ as a sum of terms involving the $H^{*-|J|}\left(K_{J}, \partial K_{J}\right)$, where $\partial K_{J} \cong \operatorname{Lk}(J)$. In the calculation of $L^{2}$ cohomology in Theorem 3.7 only the term with $J=\emptyset$ enters. Hence, under the canonical map, $H^{*}\left(A_{L} ; \mathbb{Z} A_{L}\right) \rightarrow L^{2} H^{*}\left(A_{L}\right)$, all the terms with $J \neq \varnothing$ go to 0 . Similarly, in Theorem 4.3 we calculated $H^{*}\left(\mathrm{BB}_{L} ; \mathbb{Z} \mathrm{BB}_{L}\right)$ as a sum of terms involving $H^{*-|J|+1}\left(K_{J}, \partial K_{J}\right)$. (Since $L$ is acyclic, the term with $J=\emptyset$ does not appear.) On the other hand, in Theorem 4.4 for $L^{2} H^{*}\left(\mathrm{BB}_{L}\right)$ only the terms with $|J|=1$ occur. So the canonical map $H^{*}\left(\mathrm{BB}_{L} ; \mathbb{Z B B}{ }_{L}\right) \rightarrow L^{2} H^{*}\left(\mathrm{BB}_{L}\right)$ takes all the terms with $|J|>1$ to 0 .

Remark. The cohomology of $\mathrm{BB}_{L}$ with trivial coefficients was computed by Leary and Saadetoğlu in [29].

4.3. Graph products of infinite groups. As in the Introduction, $\left\{G_{s}\right\}_{s \in S}$ is a family of groups and $G=\prod_{\Gamma} G_{s}$ is the graph product with respect to the simplicial graph $\Gamma$. The associated flag complex is $L$. For each $J$ in $\delta(L), G_{J}$ denotes the direct product of the $G_{s}$ with $s \in J$. In this section we shall also suppose that each $G_{s}$ is infinite. Put $Y=E G$. As in Example 1.6, $E G$ is the graph product of the $\left(E G_{s}, G_{s} b_{s}\right)$. The cover $\mathcal{V}=\left\{Y_{J}\right\}_{J \in S(L)}$, where $Y_{J}=G \times_{G_{J}} E G_{J}$, is a poset of spaces structure for $E G$. Let $N$ stand for $\mathbb{Z} G$ or $\mathcal{N}_{\boldsymbol{q}}(G)$. The spectral sequence of $\S 2$ converges to $H^{*}(G ; N)$ and has $E_{2}$-term

$$
E_{2}^{j . k}=H^{i}\left(K ; \mathscr{H}^{j}(\mathcal{V})\right),
$$

where the coefficient system is given by $\mathscr{H}^{j}(\mathcal{V})(\sigma)=H^{j}\left(G_{\min (\sigma)} ; N\right)$. Once we verify that condition $(\mathrm{Z})$ holds, Lemma 2.2 , will provide the following calculations.

Theorem 4.5. Let $G$ be a graph product of groups $G_{s}$, each of which is infinite. Then

$$
\operatorname{Gr} H^{n}(G ; \mathbb{Z} G)=\bigoplus_{\substack{J \in S(L) \\ i+j=n}} H^{i}\left(K_{J}, \partial K_{J} ; H^{j}\left(G_{J} ; \mathbb{Z} G\right)\right) .
$$


(Note that $H^{j}\left(G_{J} ; \mathbb{Z} G\right)=H^{j}\left(G_{J} ; \mathbb{Z} G_{J}\right) \otimes_{G_{J}} \mathbb{Z} G$.)

Theorem 4.6. Let $G$ be a graph product of groups $G_{s}$, each of which is infinite. Then

$$
L^{2} b^{n}(G)=\sum_{\substack{J \in \mathcal{S}(L) \\ i+j=n}} b^{i}\left(K_{J}, \partial K_{J}\right) \cdot L^{2} b^{j}\left(G_{J}\right) .
$$

To establish Theorem 4.5 we need to verify conditions $\left(Z^{\prime}\right)$ and $(Z)$ (or in fact just condition (Z)) which precedes Lemma 2.2. These conditions follow from statements (i) and (ii), respectively, in the next lemma.

Lemma 4.7. Suppose that $G_{J}=\prod_{s \in J} G_{s}$ is the direct product of a collection of infinite groups indexed by a finite set $J$. Let $\left\{Y_{s}\right\}_{s \in J}$ be a collection of connected CW complexes with proper $G_{s}$-actions such that each $Y_{s}$ contains a free orbit, $G_{s} b_{s}$, and put $Y_{J}:=\prod_{s \in J} Y_{s}$. As in $\S 1.4$, for each $I \subset J$, define

$$
Y_{I}^{\prime}:=\prod_{s \in I} Y_{s} \times \prod_{s \in J-I} G_{s} b_{s}
$$

Let $N$ stand for either $\mathbb{Z} G_{J}$ or $\mathcal{N}\left(G_{J}\right)$. Then:

(i) The map induced by inclusion, $H_{G_{J}}^{*}\left(Y_{J} ; N\right) \rightarrow H_{G_{J}}^{*}\left(Y_{I}^{\prime} ; N\right)$, is the zero map.

(ii) More generally, if

$$
Y_{<J}:=\bigcup_{s \in J} Y_{J-s}^{\prime},
$$

then the map induced by inclusion, $H_{G_{J}}^{*}\left(Y_{J} ; N\right) \rightarrow H_{G_{J}}^{*}\left(Y_{<J} ; N\right)$, is the zero map.

Proof. We shall prove this only in the case $N=\mathbb{Z} G_{J}$, the case $N=\mathcal{N}\left(G_{J}\right)$ being entirely similar. The relative version of the Künneth Formula states that for pairs of spaces $(A, B)$ and $\left(A^{\prime}, B^{\prime}\right)$,

$$
H^{n}\left((A, B) \times\left(A^{\prime}, B^{\prime}\right)\right)=\bigoplus_{i+j=n} H^{i}\left(A, B ; H^{j}\left(A^{\prime}, B^{\prime}\right)\right)
$$

(where $\left.(A, B) \times\left(A^{\prime}, B^{\prime}\right)=\left(A \times A^{\prime},\left(A \times B^{\prime}\right) \cup\left(B \times A^{\prime}\right)\right)\right)$. Similarly, if $(A, B)$ is a pair of $H$-spaces and $\left(A^{\prime}, B^{\prime}\right)$ a pair of $H^{\prime}$-spaces, then for left $H$-and $H^{\prime}$-modules $M$ and $M^{\prime}$,

$$
H_{H \times H^{\prime}}^{n}\left((A, B) \times\left(A^{\prime}, B^{\prime}\right) ; M \otimes M^{\prime}\right)=\bigoplus_{i+j=n} H_{H}^{i}\left(A, B ; M \otimes H_{H^{\prime}}^{j}\left(A^{\prime}, B^{\prime} ; M^{\prime}\right)\right) .
$$

By the exact sequence of the pair, showing $H_{G_{J}}^{*}\left(Y_{J} ; \mathbb{Z} G_{J}\right) \rightarrow H_{G_{J}}^{*}\left(Y_{I}^{\prime} ; \mathbb{Z} G_{J}\right)$ is zero is equivalent to showing that

$$
H_{G_{J}}^{*}\left(Y_{I} \times\left(Y_{J-I}, G_{J-I} b\right) ; M \otimes M^{\prime}\right) \rightarrow H_{G_{J}}^{*}\left(Y_{I} \times Y_{J-I} ; M \otimes M^{\prime}\right)
$$


is onto, where $M=\mathbb{Z} G_{I}, M^{\prime}=\mathbb{Z} G_{J-I}$ and $b \in Y_{J-I}$ is a basepoint. If $J-$ $I \neq \emptyset$, then, since $G_{J-I}$ is infinite and acts properly, $Y_{J-I}$ is noncompact; hence, $H_{G_{J-I}}^{0}\left(Y_{J-I} ; M^{\prime}\right)=0$ and so

$$
H_{G_{J-I}}^{j}\left(Y_{J-I}, G_{J-I} b ; M^{\prime}\right) \rightarrow H_{G_{J-I}}^{j}\left(Y_{J-I} ; M^{\prime}\right)
$$

is onto. Hence,

$$
H_{G_{I}}^{i}\left(Y_{I} ; M \otimes H_{G_{J-I}}^{j}\left(Y_{J-I}, G_{J-I} b ; M^{\prime}\right)\right) \rightarrow H_{G_{I}}^{i}\left(Y_{I} ; M \otimes H_{G_{J-I}}^{j}\left(Y_{J-I} ; M^{\prime}\right)\right)
$$

is onto. It follows from the relative Künneth Formula that the map in (4.1) is onto.

The proof of the second statement is similar using induction on the cardinality of $J$. Choose $s \in J$. Then $Y_{<J}=\left(Y_{<(J-s)} \times Y_{s}\right) \cup\left(Y_{J-s} \times G_{s} b_{s}\right)$. Hence,

$$
\left(Y_{J}, Y_{<J}\right)=\left(Y_{J-s}, Y_{<(J-s)}\right) \times\left(Y_{s}, G_{s} b_{s}\right) .
$$

Let $M=\mathbb{Z} G_{J-s}$ and $M^{\prime}=\mathbb{Z} G_{s}$. Then

$$
H_{G_{s}}^{j}\left(Y_{s}, G_{s} b_{s} ; M^{\prime}\right) \rightarrow H_{G_{s}}^{j}\left(Y_{s} ; M^{\prime}\right)
$$

is onto by the argument in the previous paragraph, and

$$
H_{G_{J-s}}^{i}\left(Y_{J-s}, Y_{<(J-s)} ; M\right) \rightarrow H_{G_{J-s}}^{i}\left(Y_{J-s} ; M\right)
$$

is onto by inductive hypothesis. Combining these two surjections, we see that

$$
\begin{gathered}
H_{G_{J-s}}^{i}\left(Y_{J-s}, Y_{<(J-s)} ; M \otimes H_{G_{s}}^{j}\left(Y_{s}, G_{s} b_{s} ; M^{\prime}\right)\right) \\
\rightarrow H_{G_{J-s}}^{i}\left(Y_{J-s} ; M \otimes H_{G_{s}}^{j}\left(Y_{s} ; M^{\prime}\right)\right)
\end{gathered}
$$

is onto. So, by the relative Künneth Formula,

$$
H_{G_{J-s} \times G_{s}}^{n}\left(\left(Y_{J-s}, Y_{<(J-s)}\right) \times\left(Y_{s}, G_{s} b_{s}\right) ; \mathbb{Z} G_{J}\right) \rightarrow H_{G_{J-s} \times G_{s}}^{n}\left(Y_{J-s} \times Y_{s} ; \mathbb{Z} G_{J}\right)
$$

is onto, which completes the proof.

Other coefficients. As before, $G=\prod_{\Gamma} G_{s}$ is the graph product and $G^{\prime}=\prod_{s \in S} G_{s}$ is the direct product. Let $p: G \rightarrow G^{\prime}$ be the natural projection. We say that a group $H$ lies between $G$ and $G^{\prime}$ if $H=G / N$ for some normal subgroup $N \subset G$ with Ker $p \subseteq N$. If this is the case, then $p$ factors as

$$
G \stackrel{f}{\rightarrow} H \rightarrow G^{\prime}
$$

where $f$ is the natural epimorphism. In this way $\mathbb{Z} H$ becomes a $G$-module. There is the following generalization of Theorem 4.5. 
Theorem 4.8. Suppose that each $G_{s}$ is infinite and $H$ lies between $G$ and $G^{\prime}$. Then

$$
\operatorname{Gr} H^{n}(G ; \mathbb{Z} H)=\bigoplus_{\substack{J \in S(L) \\ i+j=n}} H^{i}\left(K_{J}, \partial K_{J} ; H^{j}\left(G_{J} ; \mathbb{Z} H\right)\right)
$$

where as before, $H^{j}\left(G_{J} ; \mathbb{Z} H\right)=H^{j}\left(G_{J} ; \mathbb{Z} G_{J}\right) \otimes_{G_{J}} \mathbb{Z} H$. Similarly,

$$
L^{2} b^{n}((E G) / N ; H)=\sum_{\substack{U \in S(L) \\ i+j=n}} b^{i}\left(K_{J}, \partial K_{J}\right) \cdot L^{2} b^{j}\left(G_{J}\right) .
$$

We have $H^{n}(G ; \mathbb{Z} H)=H_{H}^{n}((E G) / N)$ and $(E G) / N$ is covered by complexes of the form $H \times_{G_{J}} E G_{J}$. The proof then goes through in the same manner as that of Theorem 4.5.

\section{Graph products of Coxeter groups}

5.1. Polyhedral joins. As in $\S 1.2$ and $\S 1.4$, let $L$ be a simplicial complex with vertex set $S$. For each $s \in S$, suppose that we are given a simplicial complex $\mathscr{L}(s)$ with vertex set $T_{s}$. For each $J \in S(L)$, define $\mathscr{L}(J)$ to be the join,

$$
\mathscr{L}(J):=\underset{s \in J}{*} \mathscr{L}(s),
$$

and then define the polyhedral join of the $\mathscr{L}(s)$ with respect to $L$ by

$$
*_{L} \mathscr{L}(s):=\bigcup_{J \in \mathcal{S}(L)} \mathscr{L}(J) .
$$

To simplify notation put $\mathscr{L}:=\mathscr{W}_{L} \mathscr{L}(s)$. Here is an equivalent definition. Let $T$ denote the disjoint union

$$
T:=\bigcup_{s \in S} T_{s} .
$$

and $\pi: T \rightarrow S$ the natural projection. Any subset $I$ of $T$ can be decomposed as

$$
I=\bigcup_{s \in \pi(I)} I_{s},
$$

where $I_{s} \subseteq T_{s}$. Then $I$ is the vertex set of a simplex in $\mathscr{L}$ if and only if $\pi(I) \in S(L)$, and $I_{s} \in S(\mathscr{L}(s))$ for each $s \in S$. In other words, a simplex $I$ of $\mathscr{L}$ is determined by a simplex $J \in S(L)$ and a collection of simplices $\left\{I_{s}\right\}_{s \in J}$, where each $I_{s} \in \mathcal{S}(\mathscr{L}(s))$.

Remark. Similarly, given any family of spaces $\{X(s)\}_{s \in S}$, for each $J \in S(L)$, define $X(J)$ to be the join of the $X(s)$ and the polyhedral join, $\mathcal{*}_{L} X(s)$, to be the union of the $X(J)$ as in (5.2). 
Recall that the notion of "polyhedral product" was defined by (1.3). The proof of the next lemma is straightforward.

Lemma 5.1. The operation of applying $K$ to simplicial complexes intertwines the polyhedral join with the polyhedral product (defined in $\$ 1.4$ ), i.e.,

$$
K\left(*_{L} \mathscr{L}(s)\right)=\pi_{L}(K(\mathscr{L}(s)), \partial K(\mathscr{L}(s))) .
$$

The following lemma is a well-known consequence of the Künneth Formula.

Lemma 5.2. Given two spaces $A$ and $B$,

$$
\bar{H}^{n}(A * B)=\bigoplus_{i+j=n-1} \bar{H}^{i}\left(A ; \bar{H}^{j}(B)\right) .
$$

Also, $\bar{H}^{*}(A * B) \rightarrow \bar{H}^{*}(A)$ is the zero map whenever $B$ is nonempty. (Here $\bar{H}^{*}(-)$ means reduced cohomology. Also, we follow the convention that the reduced cohomology of the empty set is $\mathbb{Z}$ in degree -1 .)

Remark. If we take coefficients in a field $\mathbb{F}$, the formula in Lemma 5.2 reads

$$
\bar{H}^{n}(A * B ; \mathbb{F})=\bigoplus_{i+j=n-1} \bar{H}^{i}(A ; \mathbb{F}) \otimes \bar{H}^{j}(B ; \mathbb{F})
$$

Similarly, for the $J$-fold join, $X(J)$, of $\{X(s)\}_{s \in J}$,

$$
\bar{H}^{n}(X(J) ; \mathbb{F})=\underset{\sum i_{s}=n-|J|+1}{\bigoplus} \bigotimes_{s \in J} \bar{H}^{i_{s}}(X(s) ; \mathbb{F}) .
$$

Proof of Lemma 5.2. Write $C A$ and $C B$ for the cones on $A$ and $B$, respectively. Then $(C A, A) \times(C B, B)=(C A \times C B, A * B)$. Since $C A \times C B$ is contractible, $\bar{H}^{n}(A * B)=H^{n+1}((C A, A) \times(C B, B))$. Hence,

$$
\begin{aligned}
\bar{H}^{n}(A * B) & =H^{n+1}((C A, A) \times(C B, B)) \\
& =\bigoplus_{i+j+2=n+1} H^{i+1}\left(C A, A ; H^{j+1}(C B, B)\right) \\
& =\bigoplus_{i+j=n-1} \bar{H}^{i}\left(A ; \bar{H}^{j}(B)\right),
\end{aligned}
$$

where the second equation is the relative Künneth Formula. This proves the first sentence. To prove the second, note that the subspace $A * \emptyset \subset A * B$ is homotopy equivalent to $A \times C B \subset A * B$. So, by the exact sequence of the pair, we need only show $H^{*}(A * B, A \times C B) \rightarrow \bar{H}^{*}(A * B)$ is onto. We have

$$
\begin{aligned}
H^{n}(A * B, A \times C B) & =H^{n}(C A \times B, A \times B)=H^{n}((C A, A) \times B) \\
& =\bigoplus_{i+j=n} H^{i}\left(C A, A ; H^{j}(B)\right) .
\end{aligned}
$$

Since the connecting homomorphism $\bar{H}^{j}(B) \rightarrow H^{j+1}(C B, B)$ is an isomorphism, it follows that $H^{n}(A * B, A) \rightarrow \bar{H}^{n}(A * B)$ is onto. 
For any $J \in S(L)$, put

$$
\mathscr{L}(<J):=\bigcup_{s \in J} \mathscr{L}(J-s),
$$

where $\mathscr{L}(J-s)$ is defined by (5.1).

Lemma 5.3 (cf. Lemma 4.7). The map $\bar{H}^{*}(\mathscr{L}(J)) \rightarrow \bar{H}^{*}(\mathscr{L}(<J))$, induced by the inclusion, is the zero homomorphism.

Proof. The proof is by induction on the cardinality of $J$. It is trivially true for $|J|=1$. So assume $|J|>1$. We first claim that for each $s \in J, H^{*}(\mathscr{L}(J), \mathscr{L}(J-s)) \rightarrow$ $H^{*}(\mathscr{L}(<J), \mathscr{L}(J-s))$ is the zero map. We have $(\mathscr{L}(J), \mathscr{L}(J-s))=\mathscr{L}(J-s) *$ $(\mathscr{L}(s), \emptyset)$. Hence, as in Lemma 5.2,

$$
H^{n}(\mathscr{L}(J), \mathscr{L}(J-s))=\bigoplus_{i+j=n-1} \bar{H}^{i}\left(\mathscr{L}(J-s) ; H^{j}(\mathscr{L}(s))\right) .
$$

Similarly, $\mathscr{L}(<J)=(\mathscr{L}(<(J-s)) * \mathscr{L}(s)) \cup(\mathscr{L}(J-s) * \emptyset)$; so,

$$
\begin{aligned}
H^{n}(\mathscr{L}(<J), \mathscr{L}(J-s)) & =H^{n}(\mathscr{L}(<(J-s)) *(\mathscr{L}(s), \emptyset)) \\
& =\bigoplus_{i+j=n-1} \bar{H}^{i}\left(\mathscr{L}(<(J-s)) ; H^{j}(\mathscr{L}(s))\right) .
\end{aligned}
$$

By inductive hypothesis, $\bar{H}^{i}(\mathscr{L}(J-s)) \rightarrow \bar{H}^{i}(\mathscr{L}(<(J-s)))$ is zero. Comparing (5.3) and (5.4), we see that $H^{*}(\mathscr{L}(J), \mathscr{L}(J-s)) \rightarrow H^{*}(\mathscr{L}(<J), \mathscr{L}(J-s))$ is the zero map, which proves the claim. By the exact sequence of the triple, this is equivalent to the statement that $H^{*}(\mathscr{L}(J), \mathscr{L}(<J)) \rightarrow H^{*}(\mathscr{L}(J), \mathscr{L}(J-s))$ is onto. By Lemma 5.2, $H^{*}(\mathscr{L}(J), \mathscr{L}(J-s)) \rightarrow \bar{H}^{*}(\mathscr{L}(J))$ is also onto; hence, so is their composition, $H^{*}(\mathscr{L}(J), \mathscr{L}(<J)) \rightarrow \bar{H}^{*}(\mathscr{L}(J))$. But this is equivalent to the statement that $\bar{H}^{*}(\mathscr{L}(J)) \rightarrow \bar{H}^{*}(\mathscr{L}(<J))$ is zero, which is what we wanted to prove.

Put $\mathcal{K}:=K(\mathscr{L})$. We want to use the spectral sequence of $\S 2$ to compute the cohomology of $\left(\mathcal{K}, \mathcal{K}^{T-I}\right)$ for any $I \in S(\mathscr{L})$. To warm up, let us do first the case $I=\emptyset$. We note that $\mathcal{K}^{T}$ is (the barycentric subdivision of) $\mathscr{L}$ and $\mathcal{K}$ is the cone on $\mathscr{L}$. Since

$$
\mathcal{K}=\pi_{L}(K(\mathscr{L}(s)), \mathscr{L}(s)) \quad \text { and } \quad \mathscr{L}=\mathscr{*}_{L} \mathscr{L}(s),
$$

$(\mathcal{K}, \mathscr{L})$ is a pair of posets of spaces over $\mathcal{S}(L)$ (cf. (5.1) and (5.2)). Lemma 5.3 says that condition (Z) of $\$ 2$ holds; so Lemma 2.2 gives the following,

$$
\operatorname{Gr} H^{n}\left(\mathcal{K}, \mathcal{K}^{T}\right)=\bigoplus_{\substack{J \in S(L) \\ i+j=n}} H^{i}\left(K_{J}, \partial K_{J} ; H^{j}(\operatorname{Cone}(\mathscr{L}(J)), \mathscr{L}(J))\right) .
$$

Note that the term $H^{j}(\operatorname{Cone}(\mathscr{L}(J)), \mathscr{L}(J))$ can be replaced by $\bar{H}^{j-1}(\mathscr{L}(J))$. Let $F:=\left\{s \in S \mid \mathscr{L}(s)\right.$ is the simplex on $\left.T_{s}\right\}$. Note that if $J \cap F \neq \emptyset$, then the 
join $\mathscr{L}(J)$ is contractible (since one of its factors is a simplex). So in this case the coefficients in (5.5), $H^{j}$ (Cone $\left.(\mathscr{L}(J)), \mathscr{L}(J)\right)$ vanish for all $j$.

Next, fix $I \in S(\mathscr{L})$. For each $s \in S, I_{s}=I \cap T_{s}$. Define a subset $G(I)$ of $S$ by

$$
G(I):=\left\{s \in \pi(I) \cap F \mid I_{s}=T_{s}\right\} .
$$

Then $G(I)$ is the vertex set of a simplex $\sigma$ of $L$ (since $G(I) \subseteq \pi(I)$ ). Let ${ }^{I} L$ be the full subcomplex of $L$ spanned by $S-G(I)$, and let ${ }^{I} K:=K\left({ }^{I} L\right)$ be the Davis chamber. It is not hard to see that ${ }^{I} L$ is homotopy equivalent to $K^{S-G(I)}$ (see [11], Lemma A.5.5, p. 416). For each $s \in S-G(I)$, let $\mathscr{L}^{I}(s)$ denote the full subcomplex of $\mathscr{L}(s)$ spanned by $T_{s}-I_{s}$ and for each $J \in S\left({ }^{I} L\right)$, put

$$
\mathscr{L}^{I}(J):=\mathscr{*}_{s \in J} \mathscr{L}^{I}(s)
$$

The usual spectral sequence argument proves the following.

Theorem 5.4. With notation as above,

$$
\operatorname{Gr} H^{n}\left(\mathcal{K}, \mathcal{K}^{T-I}\right)=\bigoplus_{\substack{J \in \mathcal{S}\left(I_{L}\right) \\ J \cap F=\emptyset \\ i+j=n-1}} H^{i}\left({ }^{I} K_{J}, \partial^{I} K_{J} ; \bar{H}^{j}\left(\mathscr{L}^{I}(J)\right)\right) .
$$

There are two extreme cases of Theorem 5.4.

Corollary 5.5. Suppose that each $\mathscr{L}(s)$ is a simplex. Then

$$
H^{n}\left(\mathcal{K}, \mathcal{K}^{T-I}\right)=H^{n}\left({ }^{I} K, \partial^{I} K\right)
$$

$\left(=\bar{H}^{n-1}\left(K^{S-G(I)}\right)=\bar{H}^{n-1}\left({ }^{I} L\right)\right)$.

Proof. If each $\mathscr{L}(s)$ is a simplex, then in Theorem 5.4, $J \cap F=J$ is nonempty unless $J=\emptyset$. When $J=\emptyset, \bar{H}^{j}\left(\mathscr{L}^{I}(J)\right)$ is nonzero only for $j=-1$ and we get the formula in the corollary.

Corollary 5.6. Suppose that no $\mathscr{L}(s)$ is a simplex. Then

$$
\operatorname{Gr} H^{n}\left(\mathcal{K}, \mathcal{K}^{T-I}\right)=\bigoplus_{\substack{J \in \mathcal{S}(L) \\ i+j=n-1}} H^{i}\left(K_{J}, \partial K_{J} ; \bar{H}^{j}\left(\mathscr{L}^{I}(J)\right)\right) .
$$

Proof. The hypothesis implies $G(I)=\emptyset$; hence, ${ }^{I} L=L$ and ${ }^{I} K=K$.

5.2. Cohomology of graph products of Coxeter groups with group ring coefficients. We continue from $\S 1.4$. We are given a family of Coxeter systems $\left\{\left(V_{s}, T_{S}\right)\right\}_{s \in S}$ and we form the graph product with respect to $\Gamma$. Let $T$ denote the disjoint union of the $T_{s}$ and put $V=\prod_{\Gamma} V_{s}$. Then $(V, T)$ is (obviously) a Coxeter 
system. The projection $\pi: V \rightarrow W_{L}$ restricts to the natural projection $T \rightarrow S$ which sends $T_{s}$ to $s$. For each $s \in S$ define $\mathscr{L}(s)$ to be $L\left(V_{s}, T_{s}\right)$. Clearly,

$$
\begin{aligned}
& K(V, T)=\pi_{L(W, S)} K\left(V_{s}, T_{s}\right):=\mathcal{K}, \\
& L(V, T)=\boldsymbol{*}_{L(W, S)} \mathscr{L}(s):=\mathscr{L} .
\end{aligned}
$$

Henceforth, we write $L$ and $K$ for $L(W, S)$ and $K(W, S)$, respectively.

Notation. For each $J \in S(W, S)$, put $T(J)=\pi^{-1}(J)$ and

$$
V_{J}:=V_{\pi^{-1}(J)}=\prod_{s \in J} V_{s} .
$$

We can combine Theorem 5.4 with Theorem 3.2 to get the following calculation of $H^{*}(V ; \mathbb{Z} V)$.

\section{Theorem 5.7.}

$$
\operatorname{Gr} H^{n}(V ; \mathbb{Z} V)=\bigoplus_{I \in S(V, T)} \bigoplus_{\substack{J \in S(I L) \\ J \cap F=\emptyset \\ i+j=n}} H^{i}\left({ }^{I} K_{J}, \partial^{I} K_{J} ; \bar{H}^{j-1}\left(\mathscr{L}^{I}(J)\right)\right) \otimes \hat{A}(V)^{I} .
$$

On the other hand, in Theorem 4.5 we calculated the cohomology of an arbitrary graph product of infinite groups and in Theorem 3.5 for an arbitrary graph product of finite groups. We would like to see that these answers agree with the above in the case of Coxeter groups.

For any $J \in S(L)$, put

$$
\mathcal{I}(J):=\{I \in S(\mathscr{L}) \mid G(I)=J\} .
$$

When each $V_{s}$ is finite,

$$
\begin{array}{rlr}
\text { Gr } H^{n}(V ; \mathbb{Z} V) & =\bigoplus_{I \in \mathcal{S}(\mathscr{L})} H^{n}\left(\mathcal{K}, \mathcal{K}^{T-I}\right) \otimes \hat{A}(V)^{I} \quad \text { (by Theorem 3.2 ) } \\
& =\bigoplus_{J \in \mathcal{S}(L)} \bigoplus_{I \in \mathcal{I}(J)} \bar{H}^{n-1}\left(K^{S-J}\right) \otimes \widehat{A}(V)^{I} \quad \text { (by Corollary 5.5) } \\
& =\bigoplus_{J \in \mathcal{S}(L)} H^{n}\left(K, K^{S-J}\right) \otimes \bigoplus_{I \in \mathcal{I}(J)} \hat{A}(V)^{I},
\end{array}
$$

where (5.8) agrees with Theorem 3.5 with

$$
\widehat{A}(J):=\bigoplus_{I \in \mathcal{I}(J)} \widehat{A}(V)^{I} .
$$

Next consider the situation where all $V_{S}$ are infinite. First we consider the special case where the base complex $L$ is a simplex. 
Lemma 5.8. Suppose that $V_{J}$ is the $J$-fold product of $\left\{V_{s}\right\}_{s \in J}$, where each $V_{s}$ is infinite. For each $I \in S\left(V_{J}, T(J)\right), \mathscr{L}^{I}(J)$ denotes the $J$-fold join defined by (5.6). Then

$$
H^{n}\left(V_{J} ; \mathbb{Z} V_{J}\right)=\bigoplus_{I \in \mathcal{S}\left(V_{J}, T(J)\right)} \bar{H}^{n-1}\left(\mathscr{L}^{I}(J)\right) \otimes \widehat{A}\left(V_{J}\right)^{I}
$$

Proof. Let $\mathcal{K}(J)$ denote the Davis chamber for $\left(V_{J}, T(J)\right)$. By Theorem 3.2,

$$
H^{n}\left(V_{J} ; \mathbb{Z} V_{J}\right)=\bigoplus_{I \in \mathcal{S}\left(V_{J}, T(J)\right)} H^{n}\left(\mathcal{K}(J), \mathcal{K}^{T(J)-I}(J)\right) \otimes \widehat{A}\left(V_{J}\right)^{I} .
$$

Moreover, $\mathcal{K}^{T(J)-I}(J)$ is homotopy equivalent to $\mathscr{L}^{I}(J)$. The formula in the lemma follows.

Finally, consider the general case when each $V_{s}$ is infinite:

$$
\begin{aligned}
\mathrm{Gr} H^{n}(V ; \mathbb{Z} V) & =\bigoplus_{I \in \mathcal{S}(V, T)} H^{n}\left(\mathcal{K}, \mathcal{K}^{T-I}\right) \otimes \widehat{A}(V)^{I} \\
& =\bigoplus_{I \in \mathcal{S}(V, T)} \bigoplus_{\substack{J \in \mathcal{S}(W, S) \\
i+j=n}} H^{i}\left(K_{J}, \partial K_{J} ; \bar{H}^{j-1}\left(\mathscr{L}^{I}(J)\right)\right) \otimes \widehat{A}(V)^{I} \\
& =\bigoplus_{\substack{J \in S(W, S) \\
i+j=n}} H^{i}\left(K_{J}, \partial K_{J} ; \bigoplus_{I \in \mathcal{S}(V, T)} \bar{H}^{j-1}\left(\mathscr{L}^{I}(J)\right) \otimes \widehat{A}(V)^{I}\right) \\
& =\bigoplus_{\substack{J \in \mathcal{S}(W, S) \\
++j=n}} H^{i}\left(K_{J}, \partial K_{J} ; H^{j}(V ; \mathbb{Z} V)\right),
\end{aligned}
$$

where the first equation follows from Theorem 3.2, the second from Corollary 5.6, the third from the fact that $\widehat{A}(V)^{I}$ is free abelian, and the last from Lemma 5.8. Moreover, (5.9) agrees with Theorem 4.5.

\section{Weighted $L^{2}$-cohomology of buildings and Coxeter groups}

6.1. Hecke-von Neumann algebras. Suppose that we are given a Coxeter system $(W, S)$ and a function $i: S \rightarrow I$ to an index set $I$ such that $i(s)=i(t)$ whenever $s$ and $t$ are conjugate in $W$. A multiparameter for $(W, S)$ is an $I$-tuple $t=\left(t_{i}\right)_{i \in I}$ of indeterminates (or of numbers). Write $t_{s}$ instead of $t_{i(s)}$. If $s_{1} \ldots s_{n}$ is a reduced expression for an element $w \in W$, then the monomial

$$
\boldsymbol{t}_{w}:=t_{s_{1}} \ldots t_{s_{n}}
$$

depends only on $w$ and not on the choice of reduced expression for it. (This follows from Tits' solution to the Word Problem for Coxeter groups, cf. [11], p. 315.) The growth series of $W$ is a power series in $t$ defined by

$$
W(\boldsymbol{t}):=\sum_{w \in W} \boldsymbol{t}_{w}
$$


This power series has a region of convergence $\mathcal{R}(W)$ (a subset of $\mathbb{C}^{I}$ ). If $W$ is finite, then $W(\boldsymbol{t})$ is a polynomial. For any Coxeter group $W$, it can be shown that $W(\boldsymbol{t})$ is a rational function of $\boldsymbol{t}$ (cf. [11], Corollary 17.1.6).

For any set $X, \mathbb{R}^{X}$ denotes the vector space of finitely supported real-valued functions on $X$. For each $x \in X, e_{x}$ denotes the indicator function of $\{x\}$ so that $\left\{e_{x}\right\}_{x \in X}$ is the standard basis for $\mathbb{R}^{X}$. For a multiparameter $\boldsymbol{q}$ of positive real numbers, define a inner product $\langle,\rangle_{\boldsymbol{q}}$ on $\mathbb{R}^{W}$ by

$$
\left\langle e_{w}, e_{w^{\prime}}\right\rangle_{\boldsymbol{q}}= \begin{cases}\boldsymbol{q}_{w} & \text { if } w=w^{\prime} \\ 0 & \text { otherwise }\end{cases}
$$

Let $L_{\boldsymbol{q}}^{2}(W)$ denote the Hilbert space completion of $\mathbb{R}^{W}$ with respect to $\langle,\rangle_{\boldsymbol{q}}$.

Using $\boldsymbol{q}$, one can also give $\mathbb{R}^{W}$ the structure of a Hecke algebra, determined by the formula

$$
e_{s} e_{w}= \begin{cases}e_{s w} & \text { if } l(s w)>l(w), \\ q_{s} e_{s w}+\left(q_{s}-1\right) e_{w} & \text { if } l(s w)<l(w) .\end{cases}
$$

When $\boldsymbol{q}=\mathbf{1}$ (the multiparameter which is identically 1 ), $\mathbb{R}_{\boldsymbol{q}}(W)$ is the group algebra of $W$.

Define an anti-involution $*$ on $\mathbb{R}_{\boldsymbol{q}} W$, by $\left(\sum x_{w} e_{w}\right)^{*}:=\sum x_{w^{-1}} e_{w}$. The inner product $\langle,\rangle_{\boldsymbol{q}}$ and the anti-involution $*$ give $\mathbb{R}_{\boldsymbol{q}} W$ the structure of a Hilbert algebra (see [21], Proposition 2.1). (In other words, $x^{*}$, the image of $x$ under the anti-involution of algebras, is equal to the adjoint of $x$ with respect to $\langle,\rangle_{\boldsymbol{q}}$.) This implies that there is an associated von Neumann algebra $\mathcal{N}_{\boldsymbol{q}}(W)$ (called the Hecke-von Neumann algebra) acting from the right on $L_{\boldsymbol{q}}^{2}(W)$. One definition of $\mathcal{N}_{\boldsymbol{q}}(W)$ is that it is the algebra of all bounded linear endomorphisms of $L_{\boldsymbol{q}}^{2}(W)$ which commute with the left $\mathbb{R}_{\boldsymbol{q}}(W)$-action. An equivalent definition is that it is the weak closure of the elements $\mathbb{R}_{\boldsymbol{q}}(W)$ which act from the right on $L_{\boldsymbol{q}}^{2}(W)$ as bounded linear operators.

Define the von Neumann trace of $\varphi \in \mathcal{N}_{\boldsymbol{q}}(W)$ by $\operatorname{tr}_{\mathcal{N}_{\boldsymbol{q}}}(\varphi):=\left\langle e_{1} \varphi, e_{1}\right\rangle_{\boldsymbol{q}}$ and similarly, for any $(n \times n)$-matrix with coefficients in $\mathcal{N}_{\boldsymbol{q}}(W)$. This allows us to define the von Neumann dimension of any closed subspace of an $n$-fold orthogonal direct sum of copies of $L_{\boldsymbol{q}}^{2}(W)$ which is stable under the diagonal $\mathbb{R}_{\boldsymbol{q}}(W)$-action: if $V \subset\left(L_{\boldsymbol{q}}^{2}(W)\right)^{n}$ is such a subspace and $p_{V}:\left(L_{\boldsymbol{q}}^{2}(W)\right)^{n} \rightarrow\left(L_{\boldsymbol{q}}^{2}(W)\right)^{n}$ is orthogonal projection onto $V$, then $p_{v} \in \mathcal{N}_{\boldsymbol{q}}(W)$, so define

$$
\operatorname{dim}_{\mathcal{N}_{\boldsymbol{q}}} V:=\operatorname{tr}_{\mathcal{N}_{\boldsymbol{q}}}\left(p_{V}\right) .
$$

For any $J \subset S$ and $\boldsymbol{q} \in \mathcal{R}\left(W_{J}\right)$, there is a self-adjoint idempotent $a_{\boldsymbol{J}} \in \mathcal{N}_{\boldsymbol{q}}(W)$ defined by

$$
a_{J}:=\frac{1}{W_{J}(\boldsymbol{q})} \sum_{w \in W_{J}} e_{w}
$$


(cf. [11], Lemma 19.2.5). For $s \in S$, write $a_{s}$ instead of $a_{\{s\}}$. For $s \in S$ and $J \subset S$, define subspaces of $L_{\boldsymbol{q}}^{2}(W)$ by

$$
A^{s}:=L_{\boldsymbol{q}}^{2}(W) a_{s}, \quad A^{J}:=\bigcap_{s \in J} A^{s} .
$$

These subspaces are stable under the action of $\mathbb{R}_{\boldsymbol{q}} W$ from the left. Moreover, $A^{J}$ is the image of the idempotent $a_{J}$ if $\boldsymbol{q} \in \mathcal{R}\left(W_{J}\right)$, and $A^{J}=0$ whenever $\boldsymbol{q} \notin \mathcal{R}\left(W_{J}\right)$ (cf. [11], §19.2).

Let $A^{>J}$ denote the subspace $\sum_{I>J} A^{I}$ of $A^{J}$ and put

$$
D^{J}:=A^{J} \cap\left(A^{>J}\right)^{\perp}
$$

The following is one of the main results of [15] (or see [11], Theorem 20.6.1).

Theorem 6.1 (The Decomposition Theorem of [15]). If $\boldsymbol{q} \in \overline{\mathcal{R}} \cup \overline{\mathcal{R}^{-1}}$, then

$$
\sum_{I \supseteq J} D^{I}
$$

is a direct sum and a dense subspace of $A^{J}$. In particular, taking $J=\emptyset$,

$$
L_{\boldsymbol{q}}^{2}=\overline{\sum D^{I}}
$$

If $\boldsymbol{q} \in \overline{\mathcal{R}}$, the only nonzero terms in this sum are those with I cospherical (i.e., with $S-I \in S$ ), and if $q^{-1} \in \overline{\mathcal{R}}$, the only nonzero terms are those with I spherical. Moreover, for $\boldsymbol{q}^{-1} \in \overline{\mathcal{R}}$,

$$
\operatorname{dim}_{\mathcal{N}_{\boldsymbol{q}}} D^{J}=\sum_{I \in S_{\geq J}} \frac{(-1)^{|I-J|}}{W_{I}(\boldsymbol{q})}
$$

6.2. Weighted $L^{2}$-Betti numbers. Suppose that $M$ is a mirrored $C W$ complex over $S$. Let $\boldsymbol{p}$ be a multiparameter of positive real numbers for a Coxeter system $(W, S)$. Define a measure $\mu_{\boldsymbol{p}}$ on the set of cells of $U(W, M)$ by $\mu_{\boldsymbol{p}}(c):=\boldsymbol{p}_{w}$, where $w \in W$ is the element of shortest length which moves $c$ into the base chamber $M(=$ the image of $1 \times M$ in $U(W, M))$. As in [15] or [11], we can use $\mu_{\boldsymbol{p}}$ to define the weighted $L^{2}$-cochains, $L_{p}^{2} C^{*}(U(W, M))$. The corresponding reduced cohomology groups are denoted $L_{\boldsymbol{p}}^{2} H^{*}(\mathcal{U}(W, M))$. The weighted cochains on $\mathcal{U}(W, M)$ can also be regarded as cochains on $M$ with respect to a certain coefficient system $\mathcal{I}\left(L_{\boldsymbol{p}}^{2}\right)$. This coefficient system associates to a cell $c$ in $M$, the left $\mathcal{N}_{\boldsymbol{p}}$-module, $L_{\boldsymbol{p}}^{2}(W) a_{S(c)}$, i.e.,

$$
\mathcal{I}\left(L_{p}^{2}\right)(c):=L_{p}^{2}(W) a_{S(c)}
$$


The corresponding cochain complex is denoted $C^{*}\left(M ; \mathfrak{I}\left(L_{p}^{2}\right)\right)$ and its reduced cohomology by $H^{*}\left(M ; \mathcal{I}\left(L_{\boldsymbol{p}}^{2}\right)\right)$. We have natural identifications,

$$
\begin{aligned}
& C^{*}\left(M ; \mathcal{I}\left(L_{\boldsymbol{p}}^{2}\right)\right) \cong L_{\boldsymbol{p}}^{2} C^{*}(U(W, M)), \\
& H^{*}\left(M ; \mathcal{I}\left(L_{\boldsymbol{p}}^{2}\right)\right) \cong L_{\boldsymbol{p}}^{2} H^{*}(U(W, M)) .
\end{aligned}
$$

(This is completely analogous to (3.1) and (3.2) of $§ 3$. See [14], [15].) The $j^{\text {th }}$ weighted $L^{2}$-Betti number of $U(W, M)$ is defined by

$$
L_{\boldsymbol{p}}^{2} b^{j}(U(W, M)):=\operatorname{dim}_{\mathcal{N}_{\boldsymbol{p}}} L_{\boldsymbol{p}}^{2} H^{j}(U(W, M))=\operatorname{dim}_{\mathcal{N}_{\boldsymbol{p}}} H^{j}\left(M ; \mathcal{I}\left(L_{\boldsymbol{p}}^{2}\right)\right),
$$

where $\operatorname{dim}_{\mathcal{N}_{\boldsymbol{p}}}$ is defined by (6.1). Also, put

$$
L_{\boldsymbol{p}}^{2} b^{j}(W):=L_{\boldsymbol{p}}^{2} b^{j}(U(W, K)) .
$$

We recall some of the main results of [15] and [21].

Theorem 6.2 (Dymara [21]). Suppose that $\boldsymbol{p} \in \overline{\mathcal{R}}$. Then $L_{\boldsymbol{p}}^{2} b^{j}(W)=0$ for $j>0$, while

$$
L_{\boldsymbol{p}}^{2} b^{0}(W)=\operatorname{dim}_{\mathcal{N}_{\boldsymbol{p}}} A^{S}=\frac{1}{W(\boldsymbol{p})} .
$$

Theorem 6.3 ([15], Theorem 10.3). Suppose that $\boldsymbol{p}^{-1} \in \overline{\mathcal{R}}$. Then

$$
L_{\boldsymbol{p}}^{2} b^{j}(\mathcal{U}(W, M))=\sum_{J \in S(W, S)} b^{j}\left(M, M^{S-J}\right) \operatorname{dim}_{\mathcal{N}_{\boldsymbol{p}}} D^{J},
$$

where the formula for $\operatorname{dim}_{\mathcal{N}_{p}} D^{J}$ is given in Theorem 6.1.

Theorem 6.4 ([15], Theorem 13.8). Suppose that $(\mathcal{C}, \delta)$ is a locally finite building of type $(W, S)$ with a chamber transitive automorphism group $G$ and that the thickness of $\mathcal{C}$ is given by a multiparameter $\boldsymbol{p}$ of integers. (In other words, each s-panel contains $p_{s}+1$ chambers.) Let $M$ be a mirrored $C W$ complex over $S$ (with a $W$-finite mirror structure). Then

$$
L^{2} b^{j}(\mathscr{B}(\mathcal{C}, M), G)=L_{\boldsymbol{p}}^{2} b^{j}(U(W, M)) .
$$

(The group $G$ need not be discrete. The von Neumann dimensions with respect to $G$ are defined using Haar measure on $G$, normalized so that the stabilizer of a chamber has measure 1.)

Corollary 6.5. Let $G$ be a graph product of finite groups $\left\{G_{s}\right\}_{s \in S}$. Let $\boldsymbol{p}$ be the multiparameter defined by $p_{s}:=\left|G_{s}\right|-1$. Then

$$
L^{2} b^{j}(G)=L_{\boldsymbol{p}}^{2} b^{j}(W) .
$$


As in the last paragraph of $\S 3$, there is a different method which can be used to define weighted $L^{2}$-Betti numbers by using ideas of Lück [30]. As in [30] there is an equivalence of categories between the category of Hilbert $\mathcal{N}_{\boldsymbol{p}}(W)$-modules (i.e., $\mathcal{N}_{\boldsymbol{p}}(W)$-stable closed subspaces of $\left.L_{\boldsymbol{p}}^{2}(W)^{n}\right)$ and the category of ordinary projective modules for $\mathcal{N}_{\boldsymbol{p}}(W)$. This allows us to define a "dimension", $\operatorname{dim}_{\mathcal{N}_{\boldsymbol{p}}} M$, of a finitely generated, projective $\mathcal{N}_{\boldsymbol{p}}(W)$-module which agrees with the dimension of the corresponding Hilbert $\mathcal{N}_{\boldsymbol{p}}(W)$-module. The $\mathcal{N}_{\boldsymbol{p}}(W)$-dimension of an arbitrary $\mathcal{N}_{\boldsymbol{p}}(W)$-module is then defined to be the dimension of its projective part.

There is a coefficient system $\mathcal{I}\left(\mathcal{N}_{\boldsymbol{p}}\right)$ on the mirrored CW complex $M$ defined by

$$
\mathcal{I}\left(\mathcal{N}_{\boldsymbol{p}}\right)(c):=\mathcal{N}_{\boldsymbol{p}}(W) a_{S(c)}
$$

The corresponding cohomology groups are denoted $H^{*}\left(M ; \mathcal{I}\left(\mathcal{N}_{\boldsymbol{p}}\right)\right)$. The dimension of $H^{j}\left(M ; \mathcal{I}\left(\mathcal{N}_{\boldsymbol{p}}\right)\right)$ is equal to that of $H^{j}\left(M ; \mathcal{I}\left(L_{\boldsymbol{p}}^{2}\right)\right)$ (and they are both equal to $j^{\text {th }}$-weighted $L_{\boldsymbol{p}}^{2}$-Betti number of $\mathcal{U}(W, M)$ ). (The advantage of using the coefficient system $\mathcal{I}\left(\mathcal{N}_{\boldsymbol{p}}\right)$ instead of $\mathcal{I}\left(L_{\boldsymbol{p}}^{2}\right)$ is that it is not necessary to use reduced cohomology and then have to keep taking closures of images.)

\section{Weighted $L^{2}$-Betti numbers of graph products of Coxeter groups}

As in $\S 5.2,\left(W_{L}, S\right)$ is the RACS associated to a graph $\Gamma,\left\{\left(V_{s}, T_{s}\right)\right\}_{s \in S}$ is a family of Coxeter systems and $(V, T)$ is the corresponding graph product of Coxeter systems. Let $\boldsymbol{q}$ be a multiparameter for $(V, T)$. It restricts to a multiparameter for each $V_{s}$, which we will denote by the same letter. By Lemma $1.1, V$ is a RAB of type $\left(W_{L}, S\right)$.

Let $\boldsymbol{p}$ be the multiparameter for $\left(W_{L}, S\right)$ given by $p_{s}=V_{s}(\boldsymbol{q})-1$. The following lemma shows that the growth series of $(V, T)$ and $\left(W_{L}, S\right)$ are related by a change of variables $\boldsymbol{q} \rightarrow \boldsymbol{p}$.

Lemma 7.1. For $w \in W_{L}$,

and therefore

$$
\sum_{v \in \pi^{-1}(w)} q_{v}=p_{w}
$$

$$
V(\boldsymbol{q})=W(\boldsymbol{p})
$$

Proof. Let $s_{1} \ldots s_{n}$ be a reduced expression for $w \in W$ and let $v \in \pi^{-1}(w)$. Then $v$ factors as a product $v_{s_{1}} \ldots v_{s_{n}}$, with $v_{s_{i}} \in V_{s_{i}}^{*}$, and this factorization gives one-to-one correspondence between $\pi^{-1}(w)$ and $V_{s_{1}}^{*} \times \cdots \times V_{s_{n}}^{*}$; moreover, $q_{v}=q_{v_{s_{1}}} \ldots q_{v_{s_{n}}}$. (Recall from $\S 1.4$ that $V_{s}^{*}=V_{s}-\{1\}$.) Hence, the growth series of $\pi^{-1}(w)$ is the product of the growth series of the $V_{s_{i}}^{*}$, and the result follows.

In the first section we compute the weighted $L^{2}$-Betti numbers of $V$ in the case where $\boldsymbol{q} \notin \mathcal{R}\left(V_{s}\right)$ for each $s \in S$. Notice that this necessarily entails that each $V_{s}$ is 
infinite. The proof uses the spectral sequence of $\S 2$ in the same way as in $\S 4.3$. In the second section we consider the opposite situation where $\boldsymbol{q} \in \overline{\mathcal{R}\left(V_{s}\right)}$ for each $s \in S$. For example, this holds for all $\boldsymbol{q}$ when each $V_{s}$ is finite. In this case the proofs are based on arguments from [15].

7.1. Large weights. In this section we assume that $\boldsymbol{q} \notin \mathcal{R}\left(V_{s}\right)$ for each $s \in S$.

\section{Theorem 7.2.}

$$
L_{\boldsymbol{q}}^{2} b^{n}(V)=\sum_{\substack{i+j=n \\ J \in S(W, S)}} b^{i}\left(K_{J}, \partial K_{J}\right) \cdot L_{\boldsymbol{q}}^{2} b^{j}\left(V_{J}\right)
$$

where

$$
L_{\boldsymbol{q}}^{2} b^{j}\left(V_{J}\right)=\prod_{\substack{\sum k(s)=k \\ s \in J}} L_{\boldsymbol{q}}^{2} b^{k(s)}\left(V_{s}\right)
$$

Proof. The proof is almost the same as the proof of Theorem 4.5. Since $(V, T)$ is a Coxeter system, we prefer to use its natural action on its Davis complex rather than on $E V$. Let $Y:=\mathcal{U}(V, \mathcal{K})$ be the Davis complex and for each $J \in S(W, S)$, put

$$
Y_{J}^{\prime}:=\mathcal{U}\left(V_{J}, \mathcal{K}(J)\right), \quad Y_{J}:=V \times_{V_{J}} Y_{J}^{\prime} .
$$

As before,

$$
Y_{J}^{\prime}=\prod_{s \in J} Y_{s}^{\prime} \quad \text { where } Y_{s}^{\prime}:=U\left(V_{s}, \mathcal{K}(s)\right) .
$$

Then $\mathcal{V}=\left\{Y_{J}\right\}_{J \in \mathcal{S}(W, S)}$ is a poset of spaces on $Y$. The spectral sequence of $\S 2$ has $E_{1}^{i, j}=C^{j}\left(K ; \mathscr{H}^{j}(\mathcal{V})\right)$, where the coefficient system is defined by $\sigma \mapsto$ $H_{V}^{j}\left(Y_{\min \sigma} ; \mathcal{N}_{\boldsymbol{q}}(V)\right)$. It converges to $H_{V}^{*}\left(Y ; \mathcal{N}_{\boldsymbol{q}}(V)\right)$ and the $\mathcal{N}_{\boldsymbol{q}}$-dimensions of these cohomology spaces are the $L_{\boldsymbol{q}}^{2}$-Betti numbers. Since $\boldsymbol{q} \notin \mathcal{R}\left(V_{s}\right)$ for each $s \in S$, $H_{V_{s}}^{0}\left(Y_{s}^{\prime} ; \mathcal{N}_{\boldsymbol{q}}\left(V_{s}\right)\right)=0$ by [21], and the relative Künneth Formula gives that

$$
H_{V_{J}}^{*}\left(Y_{J}^{\prime} ; \mathcal{N}_{\boldsymbol{q}}\left(V_{J}\right)\right) \rightarrow H_{V_{J}}^{*}\left(Y_{<J}^{\prime} ; \mathcal{N}_{\boldsymbol{q}}\left(V_{J}\right)\right)
$$

is the zero map. By Lemma 2.2,

$$
E_{2}^{i, j}=\bigoplus_{J \in \mathcal{S}(W, S)} H^{i}\left(K_{J}, \partial K_{J}\right) \otimes \operatorname{Ind}_{J}^{S}\left(\mathcal{N}_{\boldsymbol{q}}\left(V_{J}\right)\right)
$$

where $\operatorname{Ind}_{J}^{S}\left(\mathcal{N}_{\boldsymbol{q}}\left(V_{J}\right)\right)$ denotes the induced representation $\mathcal{N}_{\boldsymbol{q}}\left(V_{J}\right) \otimes_{\mathcal{N}_{\boldsymbol{q}}\left(V_{J}\right)} \mathcal{N}_{\boldsymbol{q}}(V)$, and the spectral sequence degenerates at $E_{2}$. Taking von Neumann dimensions, we get the formula for weighted $L^{2}$-Betti numbers. The last formula also follows from the Künneth Formula.

We also have a weighted version of Theorem 4.8. Let $V^{\prime}$ denote the direct sum, $\prod_{s \in S} V_{s}$. A Coxeter system $\left(V^{\prime \prime}, T\right)$ lies between $V$ and $V^{\prime}$ if its presentation is given 
by changing certain entries of its Coxeter matrix from $\infty$ to even integers $\geq 2$, more specifically, for any $\left(t_{1}, t_{2}\right)$ with $t_{i} \in T_{s_{i}}$ and $\left\{s_{1}, s_{2}\right\} \notin \operatorname{Edge}(\Gamma)$, we are allowed change $m\left(t_{1}, t_{2}\right)$ from $\infty$ to an even integer. Note that a multiparameter $\boldsymbol{q}$ for $(V, T)$ is also a multiparameter for $\left(V^{\prime}, T\right)$ and for $\left(V^{\prime \prime}, T\right)$. The proof of Theorem 7.2 also gives the following.

Theorem 7.3 (cf. Theorem 4.8).

$$
L_{\boldsymbol{q}}^{2} b^{n}\left(U\left(V^{\prime \prime}, \mathcal{K}\right)\right)=\sum_{\substack{i+j=n \\ J \in S}} b^{i}\left(K_{J}, \partial K_{J}\right) \cdot L_{\boldsymbol{q}}^{2} b^{j}\left(V_{J}\right) .
$$

7.2. Small weights. Throughout this section we suppose that the multiparameter $q$ is "small" in the sense that $\boldsymbol{q} \in \mathcal{R}\left(V_{s}\right)$ for each $s \in S$. Let $M$ be a mirrored CW complex over $S$ and let $\mathcal{B}(V, M)$ be the $M$-realization of $V$ defined by (1.1) of $\S 1.3$.

As before, define a measure $\mu_{\boldsymbol{q}}$ on the set of cells of $\mathcal{B}(V, M)$ by putting $\mu_{\boldsymbol{q}}(c):=$ $\boldsymbol{q}_{v}$, where $v \in V$ is the shortest element such that $v c$ lies in the base chamber $M$. Again, we get a cochain complex, $L_{\boldsymbol{q}}^{2} C^{*}(\mathcal{B}(V, M))$. (If $Y$ is a mirrored $\mathrm{CW}$ complex over $T$, then associated to the Coxeter system $(V, T)$ there is a different cochain complex, $L_{\boldsymbol{q}}^{2} C^{*}(U(V, Y))$.) There is a coefficient system $\mathcal{I}\left(\mathcal{N}_{\boldsymbol{q}}\right)$ on $M$ defined by

$$
\mathcal{I}\left(\mathcal{N}_{\boldsymbol{q}}\right)(c):=\mathcal{N}_{\boldsymbol{q}}(V) a_{\pi^{-1}(S(c))}
$$

and the $\mathcal{N}_{\boldsymbol{q}}(V)$-dimension of $H^{j}\left(M ; \mathcal{I}\left(\mathcal{N}_{\boldsymbol{q}}\right)\right)$ is $L_{\boldsymbol{q}}^{2} b^{j}(\mathcal{B}(V, M))$.

Let $K$ and $\mathcal{K}$ denote the geometric realizations of $\delta(W, S)$ and $\delta(V, T)$, respectively.

Theorem 7.4. $L_{\boldsymbol{q}}^{2} b^{*}(U(V, \mathcal{K}))=L_{\boldsymbol{q}}^{2} b^{*}(\mathscr{B}(V, K))$.

Proof. We use the same spectral sequence as in the proof of Theorem 7.2. It converges to $H_{V}^{*}\left(\mathcal{U}(V, \mathcal{K}) ; \mathcal{N}_{\boldsymbol{q}}(V)\right)$ and has $E_{1}$-term

$$
E_{1}^{i, j}=C^{j}\left(K ; \mathscr{H}^{j}(\mathcal{V})\right),
$$

where the coefficient system is defined by $\sigma \mapsto H_{V}^{j}\left(X_{\min \sigma} ; \mathcal{N}_{\boldsymbol{q}}(V)\right)$. Since $\boldsymbol{q} \in$ $\mathcal{R}\left(V_{s}\right)$, for each $s \in S$, by Dymara's result, Theorem 6.2, the coefficients are nonzero only for $j=0$. For $j=0$ the coefficient system is associated to the poset of coefficients $J \mapsto \mathcal{N}_{\boldsymbol{q}} a_{\pi^{-1}(J)}$. In $\S 6.2$ we denoted this coefficient system by $\mathcal{I}\left(\mathcal{N}_{\boldsymbol{q}}\right)$. So $E_{1}^{i, 0}$ is the cochain complex $C^{j}\left(K ; \mathcal{I}\left(\mathcal{N}_{\boldsymbol{q}}\right)\right)$, in other words, it is the cochain complex whose cohomology gives the $b_{\boldsymbol{q}}^{*}(\mathscr{B}(V, K))$. Thus,

$$
b_{\boldsymbol{q}}^{*}(U(V, \mathcal{K}))=b_{\boldsymbol{q}}^{*}(\mathscr{B}(V, K)) .
$$

Remark. Suppose that each $V_{s}$ is finite. Then each link $\mathscr{L}(s)$ is a simplex, and it follows that the natural map $\mathscr{L} \rightarrow L$, induced by $\pi$, has contractible fibers. It follows that $\mathcal{K}$ deformation retracts to $K$, respecting the mirror structure. This deformation retraction induces a $V$-equivariant, proper homotopy equivalence $U(V, \mathcal{K}) \rightarrow \mathcal{B}(V, K)$. So, when each $V_{s}$ is finite, Theorem 7.4 is the expected result. 
Theorem 7.5. $L_{\boldsymbol{q}}^{2} b^{*}(\mathcal{B}(V, M))=L_{\boldsymbol{p}}^{2} b^{*}(U(W, M))$.

Remark. If each $V_{s}$ is finite and $\boldsymbol{q}=\mathbf{1}$, then $V$ is a locally finite building of type $(W, S)$ of thickness $\boldsymbol{p}_{s}=\left|V_{s}\right|-1$; so this reduces to Theorem 6.4 (i.e., [15], Theorem 13.8, or [11], Theorem 20.8.4). The key point of the proof, which goes back to [21], is that the folding map $\pi$ pulls back $p$-weighted harmonic cochains to $q$-weighted harmonic cochains. The proof of Theorem 7.5 is a minor generalization of the proof of [15], Theorem 13.8, to locally infinite buildings. It occupies the end of this section.

Example 7.6. Figure 1 depicts the folding map $\pi: \mathscr{B}(V, M) \rightarrow U(W, M)$ in the case of the free product of two infinite dihedral groups. Here the graph $\Gamma$ is two disjoint points $s$ and $t$, so $W$ is $\mathbb{D}_{\infty}$ generated by $s$ and $t$. The vertex groups are also $\mathbb{D}_{\infty}$, generated by $\left\{s^{+}, s^{-}\right\}$and $\left\{t^{+}, t^{-}\right\}$, respectively. So $V=\mathbb{D}_{\infty} * \mathbb{D}_{\infty}$, and we let $M$ be a segment $K$.

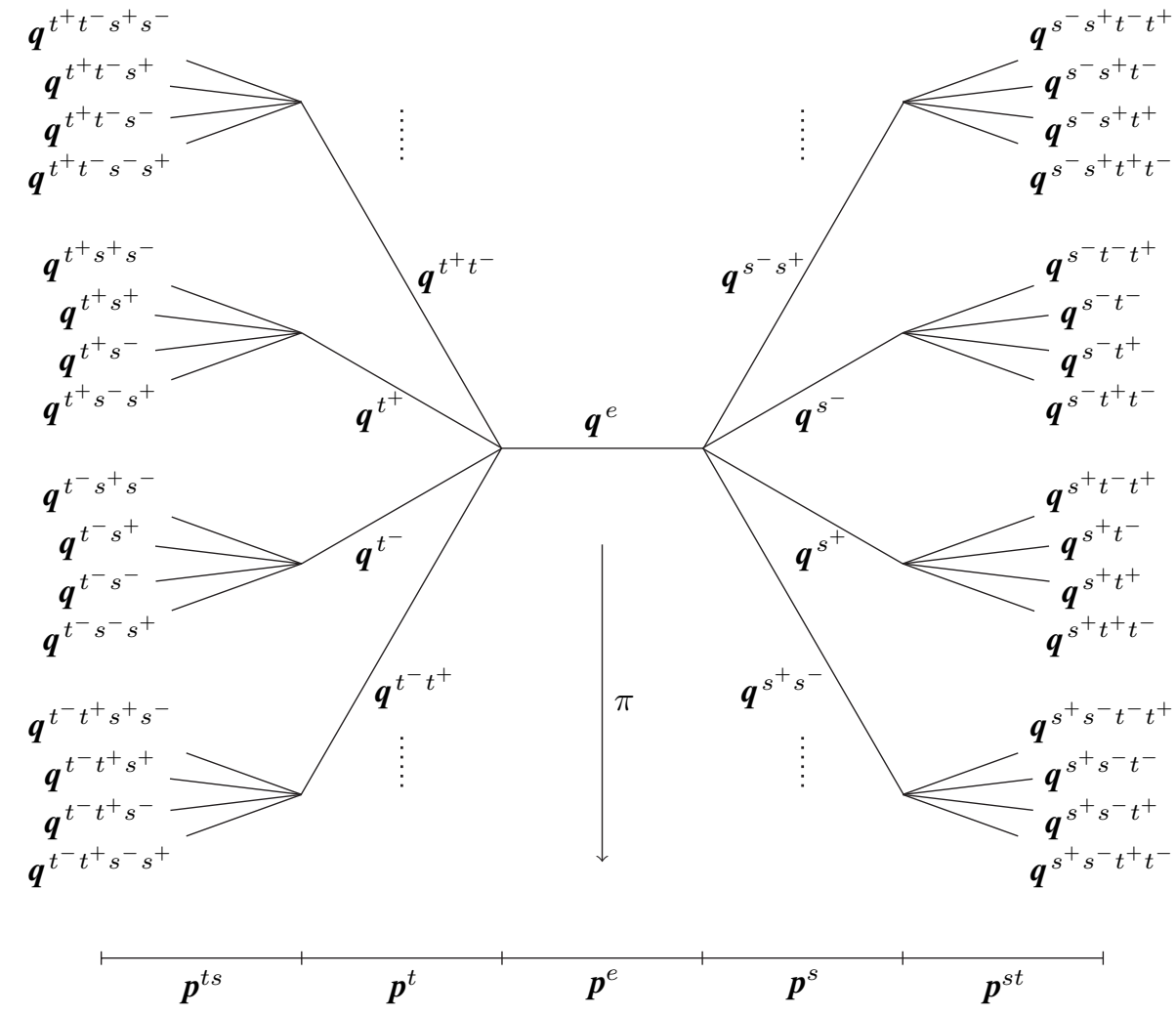

Figure 1 . $\mathbb{D}_{\infty} * \mathbb{D}_{\infty}$ as a locally infinite building over $\mathbb{D}_{\infty}$.

Combining the two previous theorems we get the following. 
Theorem 7.7. $L_{\boldsymbol{q}}^{2} b^{*}(V)=L_{\boldsymbol{p}}^{2} b^{*}(W)$.

Proof. We have

$$
\begin{aligned}
L_{\boldsymbol{q}}^{2} b^{*}(V) & :=L_{\boldsymbol{q}}^{2} b^{*}(\mathcal{U}(V, \mathcal{K}))=L_{\boldsymbol{q}}^{2} b^{*}(\mathcal{B}(V, K)) & & \text { (by Theorem 7.4) } \\
& =L_{\boldsymbol{p}}^{2} b^{*}(\mathcal{U}(W, K)) & & \text { (by Theorem 7.5) } \\
& :=L_{\boldsymbol{p}}^{2} b^{*}(W) . & &
\end{aligned}
$$

Remark 7.8 (Graph products of spherical buildings). Suppose that $\left\{\mathcal{C}_{s}\right\}_{s \in S}$ is a family of buildings where $\mathcal{C}_{s}$ is type $\left(V_{s}, T_{s}\right)$. In [12], Example 3.1(3), the first author defined the notion of a "graph product of buildings", $\prod_{\Gamma} \mathcal{C}_{s}$. It is a building of type $(V, T)$. Suppose that each $\mathscr{C}_{s}$ is spherical of thickness $\boldsymbol{q}_{s}$ with a chambertransitive automorphism group $G_{s}:=\operatorname{Aut}\left(\mathcal{C}_{s}\right)$ (i.e., each $V_{s}$ is a finite Coxeter group and the number of chambers of $\mathcal{C}_{s}$ in a panel of type $t_{s}$ is $\left.q_{t_{s}}+1\right)$. By Theorem 6.4, the ordinary $L^{2}$-Betti numbers of $\mathcal{C}:=\prod_{\Gamma} \mathcal{C}_{s}$ with respect to $G:=\prod_{\Gamma} G_{s}$ are given by

$$
L^{2} b^{j}(\mathscr{B}(\mathcal{C}, \mathcal{K}))=L_{\boldsymbol{q}}^{2} b^{j}(V)=L_{\boldsymbol{p}}^{2} b^{j}\left(W_{L}\right),
$$

where $p_{s}=V_{s}(\boldsymbol{q})-1=\left|\boldsymbol{C}_{s}\right|-1$. In other words, the $L^{2}$-Betti numbers of a graph product of spherical buildings depend only on the thickness of the buildings and the weighted $L^{2}$-Betti numbers of the associated RACS, $(W, S)$.

The proof of Theorem 7.5. The proof is a modification of the proof in [15], Theorem 13.8 in Section 13, and follows a series of lemmas.

Lemma 7.9. (i) The map $\pi: V \rightarrow W$ induces an isometric embedding

$$
\pi^{*}: L_{\boldsymbol{p}}^{2}(W) \rightarrow L_{\boldsymbol{q}}^{2}(V) .
$$

(ii) For each $s \in S, \pi^{*}\left(a_{s}\right)=a_{T_{s}}$. Moreover, for each spherical subset $J \subset S$, $\pi^{*}\left(a_{J}\right)=a_{\pi^{-1}(J)}$.

(iii) The map $\pi^{*}: L_{\boldsymbol{p}}^{2}(W) \rightarrow L_{\boldsymbol{q}}^{2}(V)$ induces a monomorphism of von Neumann algebras $\pi^{*}: \mathcal{N}_{\boldsymbol{p}}(W) \rightarrow \mathcal{N}_{\boldsymbol{q}}(V)$. (In particular, $\pi^{*}$ commutes with the $*$ antiinvolutions on $\mathcal{N}_{\boldsymbol{p}}(W)$ and $\mathcal{N}_{\boldsymbol{q}}(V)$.)

Proof. To prove (i), notice that as $w$ varies over $W$, the vectors $\pi^{*}\left(e_{w}\right)$ are orthogonal to each other, and equation (7.1) implies that $\left\|\pi^{*}\left(e_{w}\right)\right\|_{\boldsymbol{q}}=\left\|e_{w}\right\|_{\boldsymbol{p}}$.

Statement (ii) follows immediately from the definitions.

The idempotents $a_{s}$ and $a_{r}$, with $r, s \in S$, commute if and only if $r$ and $s$ commute. So, if $a_{s}$ commutes with $a_{r}$, then $a_{T_{s}}$ commutes with $a_{V_{r}}$. Since the $a_{s}$ generate the Hecke algebra, statement (iii) follows from (i) and (ii).

Similarly to the equation (7.1), the measures $\mu_{\boldsymbol{q}}$ and $\mu_{\boldsymbol{p}}$ on the cells of $\mathscr{B}(V, M)$ and $U(W, M)$ are related by

$$
\sum_{c^{\prime} \in \pi^{-1}(c)} \mu_{\boldsymbol{q}}\left(c^{\prime}\right)=\mu_{\boldsymbol{p}}(c) .
$$


By Lemma 7.9, the map $\pi: \mathscr{B}(V, M) \rightarrow \mathcal{U}(W, M)$ induces a cochain map $\pi^{*}: L_{\boldsymbol{p}}^{2} C^{*}(U(W, M)) \rightarrow L_{\boldsymbol{q}}^{2} C^{*}(\mathscr{B}(V, M))$. We also have a "transfer map" $t: L_{\boldsymbol{q}}^{2} C^{*}(\mathscr{B}(V, M)) \rightarrow L_{\boldsymbol{p}}^{2} C^{*}(\mathcal{U}(W, M))$ defined by

$$
t(f)(c):=\sum_{c^{\prime} \in \pi^{-1}(c)} f\left(c^{\prime}\right) \frac{\mu_{\boldsymbol{q}}\left(c^{\prime}\right)}{\mu_{\boldsymbol{p}}(c)} .
$$

Lemma 7.10. (i) $t \circ \pi^{*}=\mathrm{id}: L_{p}^{2} C^{i}(U(W, M)) \rightarrow L_{p}^{2} C^{i}(U(W, M))$.

(ii) The maps $\pi^{*}$ and t are adjoint to each other.

(iii) These maps take harmonic cocycles to harmonic cocycles.

Proof. Statement (i) is obvious.

(ii) For $f \in L_{\boldsymbol{p}}^{2} C^{i}(U(W, M))$ and $f^{\prime} \in L_{\boldsymbol{q}}^{2} C^{i}(\mathcal{B}(V, M))$, we have

$$
\begin{aligned}
\left\langle\pi^{*}(f), f^{\prime}\right\rangle_{\boldsymbol{q}} & =\sum_{c^{\prime} \in \mathcal{B}^{(i)}}\left[\pi^{*}(f)\left(c^{\prime}\right)\right]\left[f^{\prime}\left(c^{\prime}\right)\right] \mu_{\boldsymbol{q}}\left(c^{\prime}\right) \\
& =\sum_{c^{\prime} \in \mathcal{B}^{(i)}} f\left(\pi\left(c^{\prime}\right)\right) f^{\prime}\left(c^{\prime}\right) \mu_{\boldsymbol{q}}\left(c^{\prime}\right) \\
& =\sum_{c \in \mathcal{U}^{(i)}} f(c) \sum_{c^{\prime} \in \pi^{-1}(c)} f^{\prime}\left(c^{\prime}\right) \mu_{\boldsymbol{q}}\left(c^{\prime}\right) \\
& =\sum_{c \in \mathcal{U}^{(i)}}[f(c)]\left[t\left(f^{\prime}\right)(c)\right] \mu_{\boldsymbol{p}}(c) \\
& =\left\langle f, t\left(f^{\prime}\right)\right\rangle_{\boldsymbol{p}},
\end{aligned}
$$

where $\mathcal{B}^{(i)}$ and $\mathcal{U}^{(i)}$ denote the set of $i$-cells in $\mathcal{B}(V, M)$ and $U(W, M)$, respectively.

(iii) Since $\pi^{*}: L_{\boldsymbol{p}}^{2} C^{*}(U(W, M)) \rightarrow L_{\boldsymbol{q}}^{2} C^{*}(\mathscr{B}(V, M))$ is induced by the cellular map $\pi: \mathcal{B}(V, M) \rightarrow \mathcal{U}(W, M)$, it takes cocycles to cocycles. We must show it also takes cycles to cycles. If $c^{\prime} \in \mathscr{B}^{(i-1)}$ and $d^{\prime} \in \mathscr{B}^{(i)}$ and if the incidence number $\left[c^{\prime}: d^{\prime}\right]$ is nonzero, then it is equal to $\left[\pi\left(c^{\prime}\right): \pi\left(d^{\prime}\right)\right]$. Hence,

$$
\begin{aligned}
\partial^{\boldsymbol{q}}\left(\pi^{*}(f)\right)\left(c^{\prime}\right) & =\sum_{d^{\prime}}\left[c^{\prime}: d^{\prime}\right] \frac{\mu_{\boldsymbol{q}}\left(d^{\prime}\right)}{\mu_{\boldsymbol{q}}\left(c^{\prime}\right)} f\left(\pi\left(c^{\prime}\right)\right) \\
& =\sum_{d}[c: d] \frac{\mu_{\boldsymbol{p}}(d)}{\mu_{\boldsymbol{p}}(c)} f(c)=\partial^{\boldsymbol{p}}(f)(c),
\end{aligned}
$$

where $c=\pi\left(c^{\prime}\right), d=\pi\left(d^{\prime}\right)$, the first and the last equality come from the definition, and the middle equality comes from equation (7.2). So $\partial^{p}(f)=0$ implies that $\partial^{\boldsymbol{q}}\left(\pi^{*}(f)\right)=0$. Since $t$ is the adjoint of $\pi^{*}$, it also must take cocycles to cocycles and cycles to cycles. 
Consider the diagram

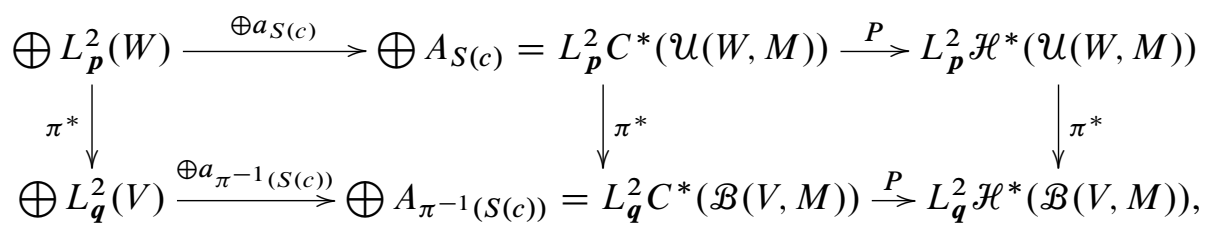

where $P$ denotes the orthogonal projection onto harmonic cocycles.

Lemma 7.11. The above diagram commutes.

Proof. The commutativity of the first square follows from Lemma 7.9.

Let $x \in L_{p}^{2} C^{*}(U(W, M))$. To prove commutativity of the second square, it is enough to show that $P \pi^{*}(x)-\pi^{*} P(x)$ is orthogonal to any harmonic cocycle $h \in L_{\boldsymbol{q}}^{2} \mathscr{H}^{*}(\mathscr{B}(V, M))$. We have: $\left\langle P \pi^{*}(x), h\right\rangle_{\boldsymbol{q}}=\left\langle\pi^{*}(x), P(h)\right\rangle_{\boldsymbol{q}}=\left\langle\pi^{*}(x), h\right\rangle_{\boldsymbol{q}}$. Hence,

$$
\left\langle P \pi^{*}(x)-\pi^{*} P(x), h\right\rangle_{\boldsymbol{q}}=\left\langle\pi^{*}(x-P(x)), h\right\rangle_{\boldsymbol{q}}=\langle x-P(x), t(h)\rangle_{\boldsymbol{p}}=0,
$$

where the second and third equalities follow, respectively, from parts (ii) and (iii) of Lemma 7.10.

Proof of Theorem 7.5. Let $e_{c} \in \bigoplus L_{\boldsymbol{q}}^{2}(V)$ denote the unit vector $e_{1} \in L_{\boldsymbol{q}}^{2}(V)$ in the summand corresponding to a cell $c \in M^{(i)}$, and similarly for $L_{\boldsymbol{p}}^{2}(W)$. Note that $\pi^{*}\left(e_{c}\right)=e_{c}$. Let $\theta$ denote the compositions of the maps in the top and the bottom rows of the above diagrams, i.e., $\theta$ is the orthogonal projection of the free Hecke-von Neumann module onto harmonic cocycles. Using Lemmas 7.11 and 7.10, we get

$$
\begin{aligned}
b_{\boldsymbol{q}}^{i}(\mathcal{B}(V, M)) & :=\operatorname{dim}_{\mathcal{N}_{\boldsymbol{q}}(V)} L_{\boldsymbol{q}}^{2} H^{i}(\mathscr{B}(V, M)) \\
& =\sum\left\langle\theta\left(e_{c}\right), e_{c}\right\rangle_{\boldsymbol{q}}=\sum\left\langle\theta \pi^{*}\left(e_{c}\right), \pi^{*}\left(e_{c}\right)\right\rangle_{\boldsymbol{q}} \\
& =\sum\left\langle\pi^{*} \theta\left(e_{c}\right), \pi^{*}\left(e_{c}\right)\right\rangle_{\boldsymbol{q}}=\sum\left\langle\theta\left(e_{c}\right), t \pi^{*}\left(e_{c}\right)\right\rangle_{\boldsymbol{p}} \\
& =\sum\left\langle\theta\left(e_{c}\right),\left(e_{c}\right)\right\rangle_{\boldsymbol{p}}=\operatorname{dim}_{\mathcal{N}_{p}(W)} L_{\boldsymbol{p}}^{2} H^{i}(U(W, M)) \\
& :=b_{\boldsymbol{p}}^{i}(U(W, M)) .
\end{aligned}
$$

\section{Octahedralization}

Suppose that $L$ is a simplicial complex. Its octahedralization, $\mathrm{O} L$, is defined as the polyhedral join

$$
\mathrm{O} L:=*_{L} S^{0}
$$

(The definition of "polyhedral join" is given in (5.1).) 
Next we work out an example which motivated most of the calculations in this paper. For each $s \in S, V_{s}$ is the infinite dihedral group with generating set, $T_{s}:=$ $\left\{s^{+}, s^{-}\right\}$. Suppose that $\left(W_{L}, S\right)$ is the RACS associated to the graph $\Gamma$ and $(V, T)$ is the graph product of the infinite dihedral groups (so that $(V, T)$ is also a RACS). By (5.7), $L(V, T)=\mathrm{O} L$. So in this special case we shall write $W_{\mathrm{O} L}$ for $V$ and $\mathrm{O} S$ for $S$ and call the RACS, $\left(W_{\mathrm{O} L}, \mathrm{OS}\right)$, the octahedralization of $(W, S)$.

Theorem 4.5 gives the following calculation of the cohomology of $W_{\mathrm{O} L}$ with group ring coefficients.

\section{Theorem 8.1.}

$$
\operatorname{Gr} H^{n}\left(W_{\mathrm{O} L} ; \mathbb{Z} W_{\mathrm{O} L}\right)=\bigoplus_{J \in \mathcal{S}(W, S)} H^{n-|J|}\left(K_{J}, \partial K_{J}\right) \otimes \mathbb{Z}\left(W_{\mathrm{O} L} / W_{\mathrm{O} J}\right) .
$$

Proof. Since $\mathbb{D}_{\infty}$ acts properly and cocompactly on $\mathbb{R}^{1}$, it is a 1 -dimensional virtual Poincaré duality group. It follows that the cohomology of $W_{\mathrm{O} J}=\left(\mathbb{D}_{\infty}\right)^{|J|}$ with group ring coefficients is given by

$$
H^{j}\left(W_{\mathrm{O} J} ; \mathbb{Z} W_{\mathrm{O} J}\right)= \begin{cases}\mathbb{Z} & \text { if } j=|J|, \\ 0 & \text { otherwise. }\end{cases}
$$

Substituting this into the formula in Theorem 4.5 gives the result.

It is proved in [16] that the cubical complex $\widetilde{T}_{L}$ is isomorphic to $U\left(W_{\mathrm{O} L}, \mathcal{K}\right)$, and that $W_{\mathrm{O} L}$ and $A_{L}$ are commensurable. Hence, Theorem 8.1 gives a calculation of $H^{*}\left(A_{L} ; \mathbb{Z} A_{L}\right)$. (In fact this was the method used by Jensen and Meier in their proof of Theorem 3.3.)

Remark. Since $\mathbb{Z} \subset \mathbb{D}_{\infty}$, there is an obvious inclusion of graph products, $A_{L} \subset W_{\mathrm{O} L}$ (cf. [26]). However, whenever $L$ is not a simplex, the image of $A_{L}$ is of infinite index in $W_{\mathrm{O} L}$. In [16] it is proved that $A_{L}$ and $W_{\mathrm{O} L}$ are both isomorphic to subgroups of index $2^{|S|}$ in a larger RACG.

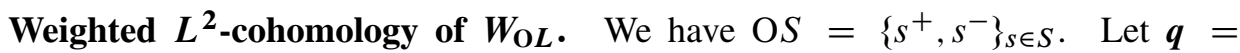
$\left(q_{s^{ \pm}}\right)_{s \in S}$. The growth series of the infinite dihedral group is easy to compute. (For example, see [11], Example 17.1.2.) We have

$$
V_{s}(\boldsymbol{q})=\frac{\left(1+q_{s^{+}}\right)\left(1+q_{s^{-}}\right)}{1-q_{s^{+}} q_{s^{-}}} \quad \text { and } \quad \frac{1}{V_{s}\left(\boldsymbol{q}^{-1}\right)}=\frac{q_{s^{+}} q_{s^{-}}-1}{\left(1+q_{s^{-}}\right)\left(1+q_{s^{+}}\right)},
$$

and

$$
p_{s}=V_{s}(\boldsymbol{q})-1=\frac{q_{s^{+}}+q_{s^{-}}+2 q_{s^{+}} q_{s^{-}}}{1-q_{s^{+}} q_{s^{-}}} .
$$

Write $\boldsymbol{q}<\mathbf{1}$ (resp. $\boldsymbol{q}>\mathbf{1}$ ) to mean that each $q_{s^{\alpha}}<1$ (resp. $>1$ ), for $\alpha \in\{+,-\}$. The following is a corollary of the results in $\$ 7$. 
Theorem 8.2. Suppose that $\left(W_{\mathrm{O} L}, \mathrm{O} S\right)$ is the octahedralization of $(W, S)$.

(i)

$$
W_{\mathrm{O} L}(\boldsymbol{q})=W(\boldsymbol{p}) .
$$

(ii) If $\boldsymbol{q}<\mathbf{1}$, then

$$
L_{\boldsymbol{q}}^{2} b^{n}\left(W_{\mathrm{O} L}\right)=L_{\boldsymbol{p}}^{2} b^{n}\left(W_{L}\right) .
$$

If, in addition, $\boldsymbol{p}^{-1} \in \overline{\mathcal{R}(W)}$ (i.e., $\boldsymbol{q}$ is sufficiently close to $\mathbf{1}$ ), then

$$
L_{\boldsymbol{q}}^{2} b^{n}\left(W_{\mathrm{O} L}\right)=\sum_{J \in \mathcal{S}(W, S)} b^{n}\left(K, K^{S-J}\right) \operatorname{dim}_{\mathcal{N}_{\boldsymbol{p}}} D^{J},
$$

where a formula for $\operatorname{dim}_{\mathcal{N}_{p}} D^{J}$ is given in Theorem 6.1 .

(iii) If $q=1 / 3$, then

$$
L_{1 / 3}^{2} b^{n}\left(W_{\mathrm{O} L}\right)=L^{2} b^{n}\left(W_{L}\right),
$$

(iv) If $\boldsymbol{q}>\mathbf{1}$, then

$$
L_{\boldsymbol{q}}^{2} b^{n}\left(W_{\mathrm{O} L}\right)=\sum_{J \in \mathcal{S}(W, S)} b^{n-|J|}\left(K_{J}, \partial K_{J}\right) \prod_{s \in J} \frac{q_{s}+q_{s^{-}}-1}{\left(1+q_{s^{-}}\right)\left(1+q_{s^{+}}\right)} .
$$

(v) If $\boldsymbol{q}=\mathbf{1}$, then

$$
L^{2} b^{n}\left(W_{\mathrm{O} L}\right)=b^{n}(K, \partial K)=\bar{b}^{n-1}(L),
$$

where $\bar{b}^{*}(-)$ refers to the reduced Betti-number.

Proof. Statements (i), (ii) and (iii) are immediate from the formula for $p_{s}$, Lemma 7.1, and Theorems 7.7 and 6.3.

The region of convergence of the dihedral group $\mathcal{R}\left(\mathbb{D}_{\infty}\right)$ is given by $q_{s}+q_{s^{-}}<1$. For a spherical $J, V_{J}$ is the $J$-fold product of $\mathbb{D}_{\infty}$. Thus, if $\boldsymbol{q}>\mathbf{1}$, then $L_{\boldsymbol{q}}^{2} H^{*}\left(V_{J}\right)$ is concentrated in degree $|J|$ and

$$
L_{\boldsymbol{q}}^{2} b^{|J|}\left(V_{J}\right)=\frac{1}{V_{J}\left(\boldsymbol{q}^{-1}\right)}=\prod_{s \in J} \frac{q_{s}+q_{s^{-}}-1}{\left(1+q_{s^{-}}\right)\left(1+q_{s^{+}}\right)} .
$$

and we apply Theorem 7.2 to obtain (iv). Finally, if $\boldsymbol{q}=\mathbf{1}$, then all the terms with nonempty $J$ in (iv) (or (ii)) vanish and we obtain (v). (Since $L^{2} b^{n}\left(W_{\mathrm{O} L}\right)=$ $L^{2} b^{n}\left(A_{L}\right)$, it also follows from Theorem 3.7.)

Remark 8.3. If $\boldsymbol{q}$ is such that $q_{s^{+}}=q_{s^{-}}\left(=q_{s}\right)$, then in Theorem 8.2 (i) the formula becomes

$$
L_{\boldsymbol{q}}^{2} b^{n}\left(W_{\mathrm{O} L}\right)=\sum_{J \in \mathcal{S}} b^{n-|J|}\left(K_{J}, \partial K_{J}\right) \prod_{s \in J} \frac{q_{s}-1}{1+q_{s}},
$$

and in (ii) the formula for $p_{s}$ simplifies to

$$
p_{s}=\frac{2 q_{s}}{1-q_{s}} .
$$


Example 8.4. This example illustrates how the formulas in Theorem 8.2 extend the calculations of [21], [15]. Suppose that $L$ is a pentagon (i.e., a circle subdivided into 5 edges). Then $\mathrm{O} L$ is a four-valent graph with 10 vertices and 20 edges. Assume $q=q$, a constant. Then $\boldsymbol{p}$ is also a constant $p$. By the previous remark $p=2 q /(1-q)$ and $q=p /(2+p)$. By [11], Example 17.1.15, the growth series are as follows:

$$
W_{L}(p)=\frac{(1+p)^{2}}{p^{2}-3 p+1}, \quad W_{\mathrm{O} L}(q)=\frac{(1+q)^{2}}{11 q^{2}-8 q+1} .
$$

The smaller roots of the denominators give the radii of convergence of these growth series as $\rho_{L}=(3-\sqrt{5}) / 2$ and $\rho_{\mathrm{O} L}=(4-\sqrt{5}) / 11$, respectively. The methods of [21], [15] give the calculation of $L_{q}^{2} b^{*}\left(W_{\mathrm{O} L}\right)$ only for $q \leq \rho_{\mathrm{O} L} \approx .16036$ or $q \geq 1 / \rho_{\mathrm{O} L}=4+\sqrt{5} \approx 6.2361$ (cf. [11], Theorem 20.7.1). However, by using Theorem 8.2 we get the answer for all $q$.

First of all, we know the weighted Betti numbers of $W_{L}$ for all $p$. They are nonzero in only one degree: for $p \leq \rho_{L}$ in degree 0 , for $\rho_{L} \leq p \leq 1 / \rho_{L}$ in degree 1 , and for $p \geq 1 / \rho_{L}$ in degree 2; moreover, in all cases the Betti number is the absolute value of $1 / W_{L}(p)$ (cf. [11], Corollary 20.4.5).

Using statement (ii) of Theorem 8.2, we get that, for $q \leq 1, L_{q}^{2} b^{*}\left(W_{L}\right)$ is concentrated, for $q \leq(4-\sqrt{5}) / 11$ in degree 0 , for $(4-\sqrt{5}) / 11 \leq q \leq(4+\sqrt{5}) / 11$ in degree 1 , and for $(4+\sqrt{5}) / 11 \leq q \leq 1$ in degree 2 ; moreover, in all cases the nonzero Betti number is $\left|1 / W_{\mathrm{O} L}(q)\right|$. For $q \geq 1$, we can use statement (iv) of Theorem 8.2 and the fact that $L_{q}^{2} b^{1}\left(\mathbb{D}_{\infty}\right)=(q-1) /(1+q)$ to see that only the second weighted Betti number is nonzero and that it is given by

$$
L_{q}^{2} b^{2}\left(W_{\mathrm{O} L}\right)=1+5\left[\frac{q-1}{1+q}\right]+5\left[\frac{q-1}{1+q}\right]^{2}=\frac{11 q^{2}-8 q+1}{(1+q)^{2}}=\frac{1}{W_{\mathrm{O} L}(q)} .
$$

Remark 8.5. Our original motivation for computing the weighted $L^{2}$-cohomology of the octahedralization of $W_{L}$ was to compute the ordinary $L^{2}$-cohomology of any Bestvina-Brady group $\mathrm{BB}_{L}$ of type $\mathrm{FP}$ (which we did by the spectral sequence method in Theorem 4.4). Although we were never able to make complete sense of the calculation, it was supposed to go something like this. Suppose that the multiparameter $\boldsymbol{q}$ is a positive constant $q$. Then it should be possible to define the weighted $L^{2}$-cohomology of $A_{L}$ and $\mathrm{BB}_{L}$. Moreover, the $L_{q}^{2}$-Betti numbers of $A_{L}$ should equal those of $W_{\mathrm{O} L}$ and $L_{q}^{2}$-Betti numbers of $\mathrm{BB}_{L}$ should behave as if the Davis complex for $W_{\mathrm{O} L}$ were to split as a product of the complex $Z_{L}$ with the real line (with $\mathbb{D}_{\infty}$-action) and as if the Künneth formula were true, i.e.,

$$
\begin{aligned}
& L_{q}^{2} b^{n}\left(W_{\mathrm{O} L}\right)=L_{q}^{2} b^{n}\left(\mathrm{BB}_{L}\right) \cdot L_{q}^{2} b^{0}\left(\mathbb{D}_{\infty}\right)=L_{q}^{2} b^{n}\left(\mathrm{BB}_{L}\right)\left[\frac{1-q}{q+1}\right] \text { for } q<1, \\
& L_{q}^{2} b^{n+1}\left(W_{\mathrm{O} L}\right)=L_{q}^{2} b^{n}\left(\mathrm{BB}_{L}\right) \cdot L_{q}^{2} b^{1}\left(\mathbb{D}_{\infty}\right)=L_{q}^{2} b^{n}\left(\mathrm{BB}_{L}\right)\left[\frac{q-1}{q+1}\right] \text { for } q>1 \text {. }
\end{aligned}
$$


These can be rewritten as

and

$$
L_{q}^{2} b^{n}\left(\mathrm{BB}_{L}\right)=L_{q}^{2} b^{n}\left(W_{\mathrm{O} L}\right)\left[\frac{q+1}{1-q}\right] \quad \text { for } q<1,
$$

$$
=L_{q}^{2} b^{n+1}\left(W_{\mathrm{O} L}\right)\left[\frac{q+1}{q-1}\right] \text { for } q>1 .
$$

Next we want to find the ordinary $L^{2}$-Betti numbers of $\mathrm{BB}_{L}$ by taking the limit of either of these formulas as $q \rightarrow 1$. Since

$$
W_{I}(p)=(1+p)^{|I|}=\left(\frac{2 q}{1-q}+1\right)^{|I|}=\left(\frac{1+q}{1-q}\right)^{|I|},
$$

the formula in Theorem 6.1 becomes

$$
\operatorname{dim}_{\mathcal{N}_{\boldsymbol{p}}} D^{J}=(-1)^{|J|} \sum_{I \in \mathcal{S}(W, S)_{\geq J}}\left(\frac{q-1}{1+q}\right)^{|I|}
$$

After multiplying through by $(1-q) /(1+q)$, the only terms on the right-hand side of (8.3) which will be nonzero when $q=1$ are those with $|I|=1$ and hence, $|J|=0$ or 1 . Since $\partial K=K^{S}$ is acyclic, the term for $J=\emptyset$ in Theorem 8.2, namely, $b^{n}\left(K, K^{S}\right)$, vanishes. So, using Theorem 8.2 (i), formula (8.1) gives, for $q<1$,

$$
L^{2} b^{n}\left(\mathrm{BB}_{L}\right)=\lim _{q \rightarrow 1} L_{q}^{2} b^{n}\left(\mathrm{BB}_{L}\right)=\sum_{s \in S} b^{n}\left(K, K^{S-s}\right)=\sum_{s \in S} b^{n}\left(K_{s}, \partial K_{s}\right),
$$

where the last equation follows from the fact that $\partial K$ is acyclic and the excision, $H^{*}\left(\partial K, K^{S-s}\right) \cong H^{*}\left(K_{s}, \partial K_{S}\right)$. Similarly, for $q>1$, Theorem 8.2 (ii) can be written as

$$
L_{q}^{2} b^{n+1}\left(W_{\mathrm{O} L}\right)=\sum_{J \in \mathcal{S}(W, S)} b^{n-|J|+1}\left(K_{J}, \partial K_{J}\right)\left(\frac{q-1}{1+q}\right)^{|J|}
$$

and (8.2) gives, for $q>1$,

$$
L^{2} b^{n}\left(\mathrm{BB}_{L}\right)=\lim _{q \rightarrow 1} L_{q}^{2} b^{n}\left(\mathrm{BB}_{L}\right)=\sum_{s \in S} b^{n}\left(K_{s}, \partial K_{S}\right),
$$

since when $q=1$ only the terms with $|J|=1$ are nonzero. So in Theorem 8.2 both the formulas (i) and (ii) give the same answer as Theorem 4.4.

\section{Duality groups}

An $m$-dimensional simplicial complex $L$ is Cohen-Macaulay if for each $J \in S(L)$, $\bar{H}^{*}(\operatorname{Lk}(J))$ is concentrated in degree $m-|J|$ and is torsion-free. For $J=\emptyset$, this 
means that $\bar{H}^{*}(L)$ is concentrated in degree $m$. It also implies that any maximal simplex $J$ has dimension $m$, since, when $J$ is maximal, $\operatorname{Lk}(J)=\emptyset$ and our convention is that $\bar{H}^{*}(\emptyset)$ is concentrated in degree -1 (where it is $=\mathbb{Z}$ ).

A group $G$ of type FP is an $n$-dimensional duality group if $H^{*}(G ; \mathbb{Z} G)$ is concentrated in degree $n$ and is torsion-free.

An immediate consequence of Corollary 8.1 is the following.

Proposition 9.1 (Brady-Meier [5] and Jensen-Meier [28]). Suppose that ( $W, S)$ is a RACS with nerve L. Then the octahedralization, $W_{\mathrm{O} L}$, is a virtual duality group if and only if $L$ is Cohen-Macaulay (and consequently, the same is true for the associated $\left.R A A G, A_{L}\right)$.

Brady and Meier asked if these conditions are equivalent for a general Artin group (Question 2 of [5]) and attributed the question to the first author of this paper. The equivalence follows immediately from Theorem 4.1 whenever the $K(\pi, 1)$ Conjecture holds for $A$. We state this as the following.

Proposition 9.2. Suppose that $X$ is the Salvetti complex associated to a Coxeter system with nerve L. Then $H^{*}(X ; \mathbb{Z} A)$ is concentrated in degree $n$ and is torsionfree if and only if $L$ is an $(n-1)$-dimensional Cohen-Macaulay complex.

(The "if" direction was also proved by Brown-Meier in [7] by using a different spectral sequence.)

Similarly, by Theorem 4.3, for Bestvina-Brady groups of type FP we have the following.

Proposition 9.3. Suppose that $L$ is an acyclic flag complex. Then $\mathrm{BB}_{L}$ is a duality group if and only if $L$ is Cohen-Macaulay. (For example, L could be an acyclic, compact manifold with boundary.)

As explained in [18], Section 6, for graph products of finite groups, Theorem 3.5 leads to a slightly different condition. A simplicial complex $L$ has punctured homology concentrated in dimension $m$ (abbreviated $\mathrm{PH}^{m}$ ) if for each closed simplex $\sigma$ of $L, \bar{H}_{*}(L-\sigma)$ is torsion free and concentrated in degree $m$. For an $m$-dimensional simplicial complex $L$, the $\mathrm{PH}^{m}$ condition implies that $L$ is Cohen-Macaulay but is not equivalent to it (cf. [18], Corollary 6.9).

Proposition 9.4 ([18], Theorem 6.2, and also cf. [25]). Let $G$ be the graph product of a collection of nontrivial finite groups. Then $G$ is a $n$-dimensional duality group if and only if $L$ is $\mathrm{PH}^{n-1}$. 


\section{References}

[1] P. Abramenko and K. S. Brown, Buildings. Grad. Texts in Math. 248, Springer, New York 2008. Zbl 1214.20033 MR 2439729

[2] A. Bahri, M. Bendersky, F. R. Cohen, and S. Gitler, Decompositions of the polyhedral product functor with applications to moment-angle complexes and related spaces. Proc. Natl. Acad. Sci. USA 106 (2009), 12241-12244. Zbl 1203.57009 MR 2539227

[3] M. Bestvina, Non-positively curved aspects of Artin groups of finite type. Geom. Topol. 3 (1999), 269-302. Zbl 0998.20034 MR 1714913

[4] M. Bestvina and N. Brady, Morse theory and finiteness properties of groups. Invent. Math. 129 (1997), 445-470. Zbl 0888.20021 MR 1465330

[5] N. Brady and J. Meier, Connectivity at infinity for right angled Artin groups. Trans. Amer. Math. Soc. 353 (2001), 117-132. Zbl 1029.20018 MR 1675166

[6] K. S. Brown, Cohomology of groups. Grad. Texts in Math. 87, Springer-Verlag, New York 1982. Zbl 0584.20036 MR 672956

[7] K. S. Brown and J. Meier, Improper actions and higher connectivity at infinity. Comment. Math. Helv. 75 (2000), 171-188. Zbl 1002.57003 MR 1760501

[8] R. Charney and M. W. Davis, The $K(\pi, 1)$-problem for hyperplane complements associated to infinite reflection groups. J. Amer. Math. Soc. 8 (1995), 597-627. Zbl 0833.51006 MR 1303028

[9] R. Charney and M. W. Davis, Finite $K(\pi, 1)$ s for Artin groups. In Prospects in topology (Princeton, NJ, 1994), Ann. of Math. Stud. 138, Princeton University Press, Princeton, NJ, 1995, 110-124. Zbl 0930.55006 MR 1368655

[10] M. W. Davis, The cohomology of a Coxeter group with group ring coefficients. Duke Math. J. 91 (1998), 297-314. Zbl 0995.20022 MR 1600586

[11] M. W. Davis, The geometry and topology of Coxeter groups. London Math. Soc. Monogr. Ser. 32, Princeton University Press, Princeton, NJ, 2008. Zbl 1142.20020 MR 2360474

[12] M. W. Davis, Examples of buildings constructed via covering spaces. Groups Geom. Dyn. 3 (2009), 279-298. Zbl 1177.51009 MR 2486800

[13] M. W. Davis, J. Dymara, T. Januszkiewicz, J. Meier, and B. Okun, Compactly supported cohomology of buildings. Comment. Math. Helv. 85 (2010), 551-582. Zbl 05736062 MR 2653692

[14] M. W. Davis, J. Dymara, T. Januszkiewicz, and B. Okun, Cohomology of Coxeter groups with group ring coefficients. II. Algebr. Geom. Topol. 6 (2006), 1289-1318. Zbl 1153.20038 MR 2253447

[15] M. W. Davis, J. Dymara, T. Januszkiewicz, and B. Okun, Weighted $L^{2}$-cohomology of Coxeter groups. Geom. Topol. 11 (2007), 47-138. Zbl 1173.20029 MR 2287919

[16] M. W. Davis and T. Januszkiewicz, Right-angled Artin groups are commensurable with right-angled Coxeter groups. J. Pure Appl. Algebra 153 (2000), 229-235. Zbl 0982.20022 MR 1783167

[17] M. W. Davis and I. J. Leary, The $l^{2}$-cohomology of Artin groups. J. London Math. Soc. (2) 68 (2003), 493-510. Zbl 1052.57003 MR 1994696 
[18] M. W. Davis and J. Meier, The topology at infinity of Coxeter groups and buildings. Comment. Math. Helv. 77 (2002), 746-766. Zbl 1022.20016 MR 1949112

[19] M. W. Davis and B. Okun, Vanishing theorems and conjectures for the $\ell^{2}$-homology of right-angled Coxeter groups. Geom. Topol. 5 (2001), 7-74. Zbl 1118.58300 MR 1812434

[20] G. Denham and A. I. Suciu, Moment-angle complexes, monomial ideals and Massey products. Pure Appl. Math. Q. 3 (2007), 25-60. Zbl 1169.13013 MR 2330154

[21] J. Dymara, Thin buildings. Geom. Topol. 10 (2006), 667-694. Zbl 1166.20301 MR 2240901

[22] J. Dymara and T. Januszkiewicz, Cohomology of buildings and their automorphism groups. Invent. Math. 150 (2002), 579-627. Zbl 1140.20308 MR 1946553

[23] A. Haefliger, Extension of complexes of groups. Ann. Inst. Fourier (Grenoble) 42 (1992), 275-311. Zbl 0762.20018 MR 1162563

[24] F. Haglund, Finite index subgroups of graph products. Geom. Dedicata 135 (2008), 167-209. Zbl 1195.20047 MR 2413337

[25] J. Harlander and H. Meinert, Higher generation subgroup sets and the virtual cohomological dimension of graph products of finite groups. J. London Math. Soc. (2) 53 (1996), 99-117. Zbl 0854.20062 MR 1362689

[26] T. Hsu and D. T. Wise, On linear and residual properties of graph products. Michigan Math. J. 46 (1999), 251-259. Zbl 0962.20016 MR 1704150

[27] T. Januszkiewicz and J. Świątkowski, Commensurability of graph products. Algebr. Geom. Topol. 1 (2001), 587-603. Zbl 0998.20029 MR 1875609

[28] C. Jensen and J. Meier, The cohomology of right-angled Artin groups with group ring coefficients. Bull. London Math. Soc. 37 (2005), 711-718. Zbl 1150.20037 MR 2164833

[29] I. J. Leary and M. Saadetoğlu, The cohomology of Bestvina-Brady groups. Groups Geom. Dyn. 5 (2011), 121-138. Zbl 05973330 MR 2763781

[30] W. Lück, $L^{2}$-invariants: theory and applications to geometry and $K$-theory. Ergeb Math. Grenzgeb. (3) 44, Springer-Verlag, Berlin 2002. Zbl 1009.55001 MR 1926649

[31] C. C. Squier, The homological algebra of Artin groups. Math. Scand. 75 (1994), 5-43. Zbl 0839.20065 MR 1308935

Received February 12, 2010; revised January 31, 2011

M. W. Davis, Department of Mathematics, Ohio State University, 231 W. 18th Ave.,

Columbus, OH 43210, U.S.A

E-mail: mdavis@math.ohio-state.edu

B. Okun, Department of Mathematical Sciences, University of Wisconsin-Milwaukee, P.O. Box 413, Milwaukee, WI 53201-0413, U.S.A.

E-mail: okun@uwm.edu 